\title{
Current perspectives of the signaling pathways directing neural crest induction
}

\author{
Timothy J. Stuhlmiller • Martín I. García-Castro
}

Received: 16 December 2011/Revised: 12 March 2012/ Accepted: 2 April 2012/Published online: 1 May 2012

(C) The Author(s) 2012. This article is published with open access at Springerlink.com

\begin{abstract}
The neural crest is a migratory population of embryonic cells with a tremendous potential to differentiate and contribute to nearly every organ system in the adult body. Over the past two decades, an incredible amount of research has given us a reasonable understanding of how these cells are generated. Neural crest induction involves the combinatorial input of multiple signaling pathways and transcription factors, and is thought to occur in two phases from gastrulation to neurulation. In the first phase, FGF and Wnt signaling induce NC progenitors at the border of the neural plate, activating the expression of members of the Msx, Pax, and Zic families, among others. In the second phase, BMP, Wnt, and Notch signaling maintain these progenitors and bring about the expression of definitive $\mathrm{NC}$ markers including Snail2, FoxD3, and Sox9/10. In recent years, additional signaling molecules and modulators of these pathways have been uncovered, creating an increasingly complex regulatory network. In this work, we provide a comprehensive review of the major signaling pathways that participate in neural crest induction, with a focus on recent developments and current perspectives. We provide a simplified model of early neural crest development and stress similarities and differences between four major model organisms: Xenopus, chick, zebrafish, and mouse.
\end{abstract}

Keywords Neural plate border · FGF - BMP . Wnt $\cdot$ Notch $\cdot$ MAPK $\cdot$ Smad $\cdot \beta$-Catenin

T. J. Stuhlmiller · M. I. García-Castro ( $\square)$ Department of Molecular, Cellular, and Developmental Biology, Yale University, PO Box 208103, New Haven, CT 06520-8103, USA

e-mail: martin.garcia-castro@yale.edu

\author{
Abbreviations \\ NC Neural crest \\ NPB Neural plate border \\ NP Neural plate \\ NNE Non-neural ectoderm \\ pNC Prospective neural crest \\ pNP Prospective neural plate \\ DLMZ Dorsolateral marginal zone \\ ES Embryonic stem \\ EpiSC Epiblast stem cell \\ hESCs Human embryonic stem cells
}

\section{Introduction}

The neural crest $(\mathrm{NC})$ is a remarkable population of multipotent embryonic cells unique to vertebrates, which migrate from the dorsal neural tube early in development to give rise to a diverse array of derivatives, including neurons and glia of the peripheral nervous system, sympathoadrenal cells, cardiac cells, melanocytes, and most of the bone and cartilage of the face and skull. Their origin can be traced to the border of the neural plate-a region of ectoderm situated between the neural plate (NP), which gives rise to the central nervous system, and the nonneural ectoderm (NNE), which forms the epidermis. Immediately beneath the ectoderm there is a layer of mesoderm, and together with the NP and NNE, these tissues are collectively believed to contribute to the induction of the NC. As the neural plate begins to close to form the neural tube, presumptive NC cells occupy the dorsal tips of the neural plate (the neural folds), and are laterally flanked by prospective placodal ectoderm in cranial regions and by prospective epidermis in the trunk and tail. In all organisms, NC cells undergo an epithelial-to-mesenchymal 
Fig. 1 Morphogenesis and major events in early neural crest development. Images display major morphogenetic changes in the early stages of neural crest (NC) development from gastrulation to neurulation, using the chick embryo as an example. The neural plate border (NPB) and neural crest (NC) progenitors are marked by Pax7 in red. a Signaling molecules induce NC progenitors at the prospective NPB before and during the gastrula stage, but the source of inductive signals varies by organism. $\mathbf{b} \mathrm{NC}$ progenitors are first identifiable with molecular markers of the neural plate border (NPB), including Msx1/ 2, Pax3/7, Zic1, Dlx3/5, Hairy2, Id3, and Ap2. The NPB is flanked medially by the neural plate (NP) and laterally by the non-neural ectoderm (NNE), with a layer of mesoderm found underneath. At the neurula stage, signaling between these tissues maintains the expression of NPB markers. c As the NP thickens and rises, the transcriptional activity of NPB specifiers and additional signaling events lead to the expression of NC specifiers at the neural folds, including Snail2, FoxD3, Sox9/10, Twist, cMyc, and Ap2. The pre-placodal ectoderm is found immediately lateral to the $\mathrm{NC}$ in rostral regions. $\mathbf{d}$ Soon after the $\mathrm{NC}$ fate is established, NC cells undergo an epithelial-to-mesenchymal transition and migrate throughout the body and differentiate into a multitude of derivatives. In the chick, NC cells migrate soon after the neural tube fuses, but in most other organisms, NC cells begin to migrate before the neural tube is closed

transition (EMT) in a rostrocaudal wave and take on stereotypical patterns of migration and give rise to various cell types. The rostral, cranial NC cells are the first to delaminate-they begin to migrate before neural tube closure in the mouse, frog (Xenopus), and zebrafish, but cranial NC cells in the chick begin migration soon after apposition of the neural folds. At more caudal levels, trunk NC cells migrate from the dorsal aspect of the forming neural tube. The early morphogenesis of NC development is outlined in Fig. 1, using the chick embryo as an example.

$\mathrm{NC}$ development is perceived as a step-wise progression from inductive events to transcription factor expression to modulation of migration and differentiation (Fig. 1). The molecules and their interactions have been integrated into a NC gene regulatory network, providing a rich framework for continued functional and comparative research [1, 2]. Initially, the $\mathrm{NC}$ is induced by a combination of signals, most notably the bone morphogenetic protein (BMP), fibroblast growth factor (FGF), and Wnt signaling pathways, but also potentially including Notch/Delta, retinoic acid (RA), Hedgehog, and Endothelin signaling. These signaling pathways integrate early in development to induce the expression of a set of regulatory transcription factors (Msx1/2, Pax3/7, Zic1, Dlx3/5, Hairy2, Id3, Ap2), which specify the neural plate border (NPB). These factors along with combinations of the same signaling pathways then trigger the expression of NC specifiers, a second set of transcription factors including Snail2, FoxD3, Sox9/10, Twist, cMyc, and Ap2. NC specifiers are proposed to ultimately control neural crest behavior, from EMT and delamination to migration and differentiation. Because these transcription factors are expressed in other tissues at these and other times in development, their spatiotemporal
A Inductive Signaling

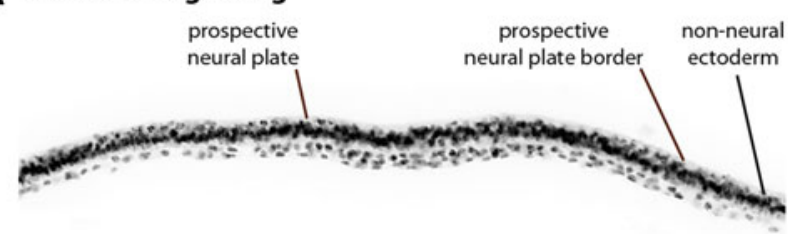

B NPB Formation and Maintenance

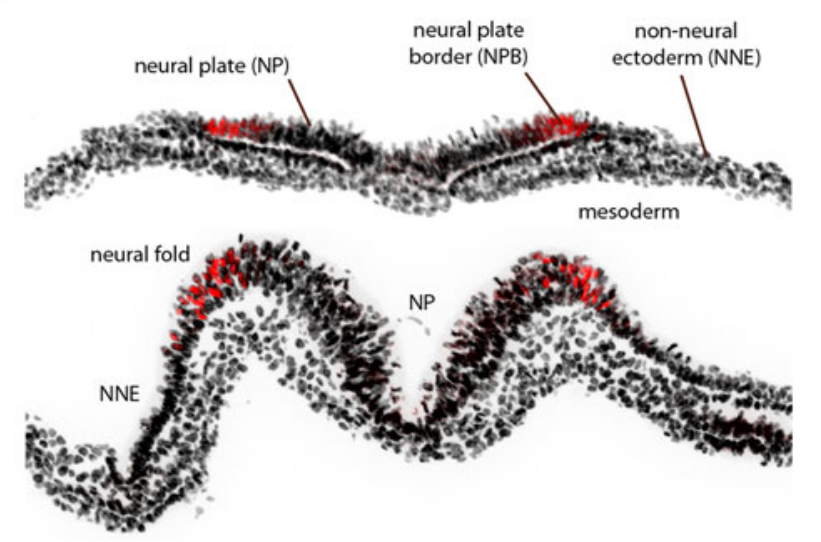

C Adoption of NC Fate

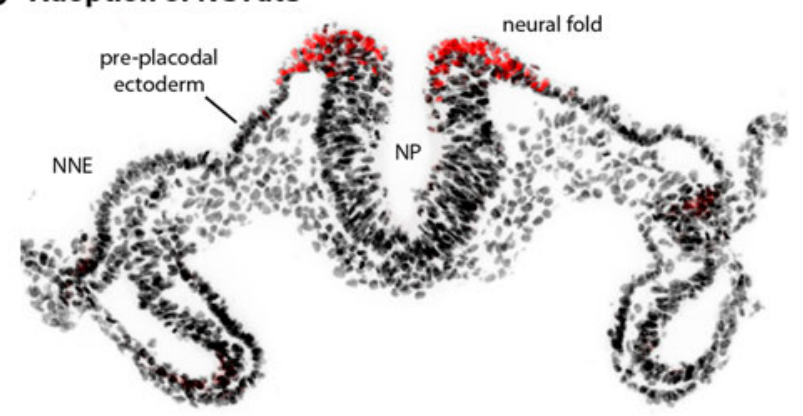

D Migration and Differentiation

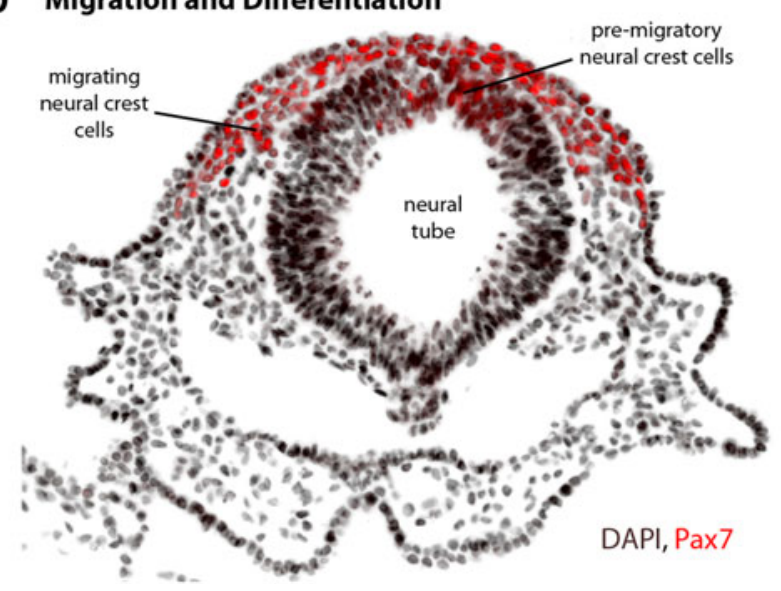

and combinatorial expression must be considered when associating them with $\mathrm{NC}$ development.

Although NC development has been studied in several species, our knowledge of the earliest inductive signaling comes primarily from Xenopus and chick research. Recent evidence from these organisms suggests that the $\mathrm{NC}$ is induced during gastrulation, and its early development can 
be explained in a two-step process. An initial phase of FGF and Wnt signaling during gastrulation induces the $\mathrm{NC}$ in the prospective NPB, and a second phase of Wnt and BMP signaling during neurulation maintains the NC population. Although the signaling pathways implicated in NC development appear to be conserved among different species, the source, timing, and precise regulation show considerable variation.

The study of early NC development has attracted significant interest owing to the unique properties of these cells. As a great model for induction, pluripotency, cellfate restrictions, migration and differentiation, $\mathrm{NC}$ development involves most elements of developmental biology. Additionally, defects in various aspects of NC development cause a number of debilitating human health conditions, collectively known as neurocristopathies, including aggressive tumors such as melanomas and neuroblastomas, rare syndromes like Hirschsprung and Waardenburg syndromes, and various developmental malformations such as cleft lip/palate and aganglionic megacolon. Therefore, NC biology is of clinical relevance as well, and a fuller understanding of the signaling mechanisms and tissue interactions giving rise to the $\mathrm{NC}$ is critical to develop better diagnostic and therapeutic tools for these disorders.

\section{Timing and transcription factors}

Neural plate border (NPB) specification and neural crest (NC) induction are mediated by a collection of ectodermally expressed regulatory transcription factors from pregastrula stages until neurulation. Markers of the NPB are currently the first molecular indication of prospective $\mathrm{NC}$ tissue and begin to be expressed during or shortly after gastrulation at a similar timepoint to the appearance of neural tissue (species-specific differences in developmental timing and tissue organization are presented in Fig. 2). Many of the genes involved in NPB formation are expressed in several other tissues and the expression and participation of a given transcription factor can vary between organisms, complicating their analyses. In Xenopus, much work has yielded a small set of transcription factors important for NPB specification, but epitasis studies demonstrate that an increasingly complex network exists. Several studies have established Msx1, Pax3, and Zic1 as crucial regulators of NPB specification [3-5], while more recently the participation of Hairy2, Gbx2, Pax7, Ap2a, and Meis3 has also been characterized [6-13]. In the chick, Pax7 is thus far the sole transcription factor implicated in regulating $\mathrm{NC}$ specifiers and is expressed exclusively in the NPB at early stages [14, 15]. Other NPB markers are expressed more broadly, with Msx 1 and Pax3 additionally expressed more caudal and lateral, Zic genes found more medial, and Ap2 expressed across the lateral NNE [16]. Interestingly, no functional studies have yet confirmed their participation in chick NC induction. In zebrafish, Msx genes are expressed at the border and are involved in NPB specification, but are not necessary for later NC markers [17-19]. Zic2a and Pax3 are expressed more highly in the NP during gastrulation and have not yet been implicated in NPB specification [20]. The expression of Ap2a and FoxD3 overlap in the prospective NC during gastrulation, and their combined activities are necessary for the earliest steps of NC induction [21]. Unique to the zebrafish, Prdm1a (Blimp1) also serves to specify the NPB fate [22-24]. In the mouse, the expression of Ap2 begins as early as E7 with Pax3/7, Msx1/2, and Zic genes becoming detectable by E7.5, about the time the neural folds form and slightly before the expression of NC specifiers and the appearance of migratory NCCs ([25-28] and our unpublished observations).

The initial expression of NC specifiers also varies across species. In Xenopus, most NC specifiers (Snail2, FoxD3, Sox $8 / 9$, others) are first expressed at stage 12 , very shortly after the appearance of the NPB, and before gastrulation has even completed [29]. In the chick, however, Snail2 is first evident at stage 6 and not strongly expressed until stage 8 (4-somite stage), several hours after the NPB has formed. Furthermore, definitive NC cells expressing a full complement of $\mathrm{NC}$ markers are not apparent until just before migration at stage 9/10 [16]. Despite these differences, the avian NC appears to be specified before gastrulation (having already received the necessary signals for differentiation when cultured in non-inducing conditions) [30], while Xenopus neural folds isolated even at the neurula stage do not maintain expression of NC markers without additional signals [31, 32]. In zebrafish, FoxD3 is expressed first and along with Ap2a has a unique role early in gastrulation [21], while Snail2, Sox9/10, and other NC specifiers label the NC towards the end of gastrulation [33]. In the mouse, NC specifiers such as Sox $9 / 10$ [34, 35] and FoxD3 [36] label the neural folds very soon after the expression of NPB markers and immediately before cranial NC migration, which is well underway by E8. Additionally, Snail1/2 seem to have switched expression domains in the mouse, but even more intriguing, a double knockout model eliminating both Snail proteins from the epiblast still generates normal, migrating NC cells [37-39]. Snail proteins are thought to be crucial regulators of both $\mathrm{NC}$ fate and EMT, and thus, other transcription factors must be providing these essential functions in the double knockout. One could envision redundancy or compensatory mechanisms, and Twist, Zeb1, and Zeb2 have been considered as candidates to provide the lost function. While possible, evidence for significant overlap between the direct targets of Snail proteins and their putative substitutes is currently 


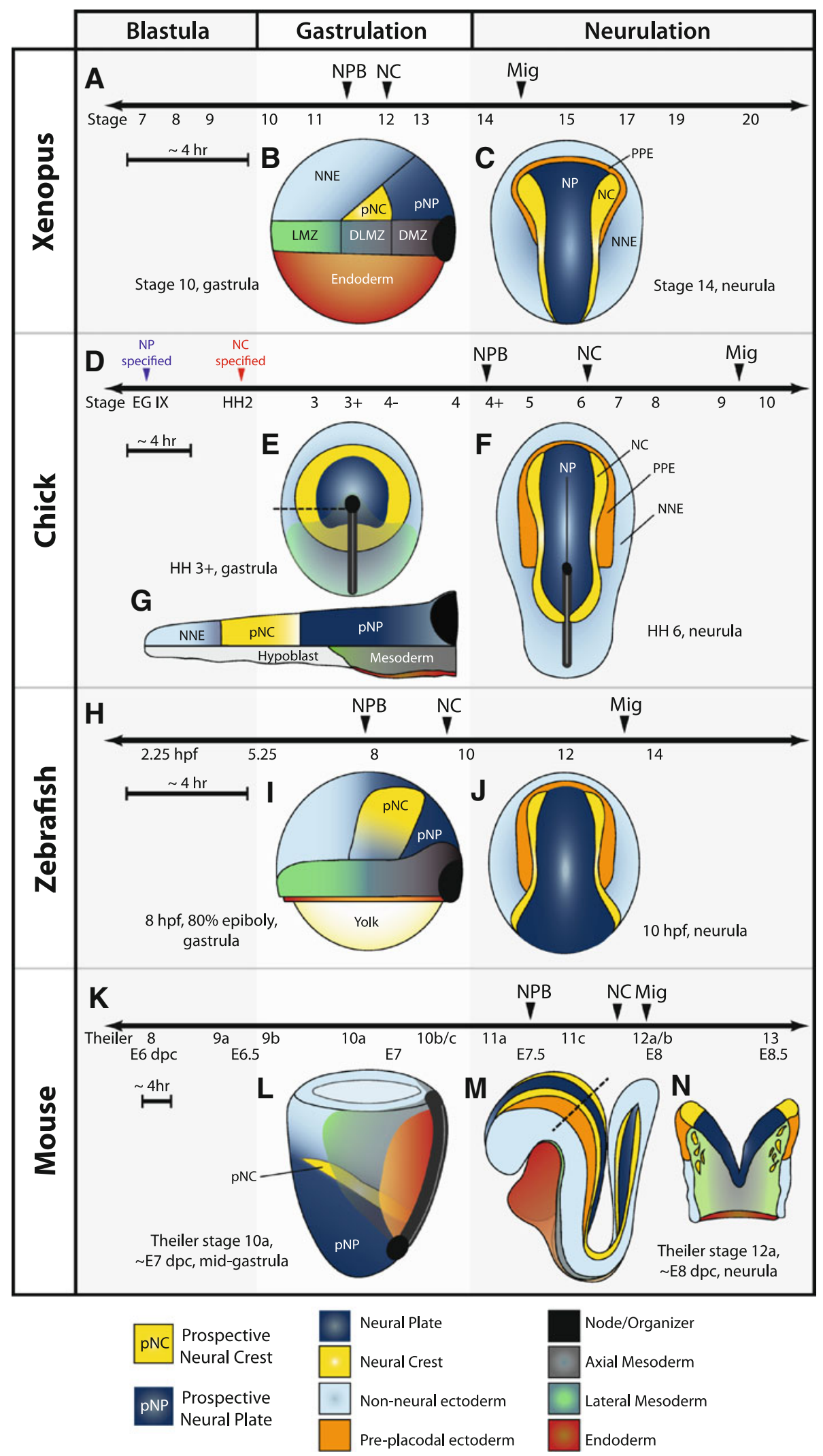

lacking. Perhaps then, the NC gene regulatory network in the mouse has diverged evolutionarily, dispensing a critical role for Snail genes in murine neural crest development.
On this note, knockdown experiments in other organisms generally cause a significant reduction of the NC markers tested, but a demonstration of a complete loss of $\mathrm{NC}$ would 
4 Fig. 2 Timing and morphology of early neural crest development in Xenopus, chick, zebrafish, and mouse. a, d, h, $\mathbf{k}$ Timelines for early events in NC development. Note the appearance of neural plate border markers (NPB) and neural crest specifiers (NC) occurs during gastrulation in anamniotes (Xenopus and zebrafish) and after gastrulation in amniotes (chick and mouse). Anamniotes progress at a higher rate of development and the time between events is generally very short-compare sizes of 4-h time bars. a In Xenopus, markers of the neural plate border are first apparent at Nieuwkoop and Faber stage 11.5 and immediately precede expression of neural crest specifiers at stage 12 , before the end of gastrulation. NC migration (Mig) begins around stage 15. b Lateral view of early Xenopus gastrula. Animal pole is up, dorsal to the right. Prospective neural crest tissue (pNC) is found above the dorsolateral marginal zone (DLMZ), based on fate-mapping studies [31]. LMZ lateral marginal zone, $D M Z$ dorsal marginal zone. c Dorsal view of a Xenopus neurula. Anterior is up. $\mathbf{d}$ In the chick, neural tissue is specified before the egg is laid at Eyal-Giladi (EG) stage IX, while neural crest tissue is specified by Hamburger and Hamilton $(\mathrm{HH})$ stage 2. Markers of the neural plate border are not apparent until after gastrulation at stage $4+$. The first neural crest specifiers are not expressed until stage 6 . Migration begins between stage 9 and 10. e Dorsal view of midgastrula. Prospective neural crest tissue is found in a ring around the prospective neural plate (pNP) until post-gastrula stages when the anterior NPB is specified to become pre-placodal ectoderm [30]. f Lateral section through the dotted line in e. At pre-gastrula and early gastrula stages, the prospective neural crest is situated above the hypoblast, an extra-embryonic tissue. As mesoderm and endoderm ingress, the hypoblast is displaced anteriorly, and by the end of gastrulation prospective neural crest tissue is underlain by mesoderm. g Dorsal view of neurula, anterior is up. NC specifiers are initially only expressed in the anterior-most aspect of the neural folds. $\mathbf{h}$ In the zebrafish, neural plate border markers and neural crest specifiers are first expressed during gastrulation. Migration occurs after $13 \mathrm{~h}$ postfertilization (hpf). i Lateral view of zebrafish gastrula. Animal pole is up, dorsal to the right. Location of prospective neural crest is inferred from expression of Msxb [70] and Pax3 [113]. j Dorsal view of neurula, anterior is up. $\mathbf{k}$ In the mouse, most neural plate border markers are first detectable around E7.5. Neural crest specifiers are expressed by E7.75, and NC cells begin migrating almost immediately after this expression. Listed below the timeline are approximate stages by Theiler stage, and embryonic days post coitum (dpc). 1 Lateral view of mouse gastrula. Anterior to the left. The mouse embryo develops with the prospective ectoderm as the interior layer. Location of the prospective neural crest is inferred from the position of prospective neural and non-neural tissues, and the expression of NPB markers by E7.5. $\mathbf{m}$ Lateral view of neurula. Anterior to the left. n Section through the dotted line in $\mathbf{~ m}$. Although the neural tube has not yet closed, NC cells are migrating extensively

require a more extensive study, and this should be considered when making comparative analyses between NC gene regulatory networks.

\section{Evidence for the inductive tissues}

$\mathrm{NC}$ formation is thought to occur by a classic induction mechanism whereby a tissue or tissues serve as source of inductive signals that are received by another tissue, resulting in the formation of a unique cell type. Pioneering work on $\mathrm{NC}$ induction implicated the mesoderm as a potential source of inductive signals_-using salamanders,
Raven and Kloos were able to generate NC derivatives by grafting presumptive paraxial and lateral plate mesoderm into the naive ectoderm of the ventral blastocoel [40]. Decades later, the inductive capacity of the mesoderm was confirmed in another amphibian, Xenopus laevis. Snail2 was induced in the naive ectoderm of the blastocoel roof following grafts of the organizer or dorsal or lateral mesoderm [41], or by co-culturing explants of naive ectoderm and the paraxial mesoderm [42, 43]. The paraxial mesoderm was subsequently shown to be required for NC formation, as its removal inhibits Snail2 expression $[42,44]$.

Other experiments instead suggested that the interaction of neural and non-neural ectoderm led to NC induction. Using two different species of amphibia, Rollhauser-ter Horst demonstrated that neural and epidermal tissues can generate NC cells when juxtaposed [45]. Using pigmented and non-pigmented axolotl embryos in donor/host combinations, Moury and Jacobson later demonstrated that NC cells could arise from both tissues [46]. Similar experiments in Xenopus and chick, grafting neural tissue into lateral epidermis, demonstrated both tissues could yield NC cells in these species as well [43, 47]. A recent Xenopus paper suggests the competence of the NNE to give rise to $\mathrm{NC}$ diminishes towards the end of gastrulation, while the NP retains its competence until neurulation [48]. Together, these findings supported a model where NC induction results from interactions between the NP, NNE, and underlying mesoderm. Recent evidence now suggests that the precise involvement of these tissues is species-specific.

In Xenopus, most current models propose that the dorsolateral marginal zone (DLMZ) of the gastrula (which underlies the prospective NC) is the source of NC-inducing signals. The DLMZ expresses multiple Wnt and FGF ligands and the BMP antagonist Chordin [31, 41, 49, 50], molecules known to be involved in NC induction. The DLMZ also expresses a number of other Wnt and BMP signaling regulators including Noggin, Cerberus, Frzb1, Dkk1, Sfrp2, and Crescent. A recent study unveils an interesting role for Snail2 in mesoderm formation and implicates this factor in regulating the signals emanating from the DLMZ, making Snail2 crucial for early events in NC development as well [51]. By neurula stages of development, the DLMZ has given rise to the paraxial or intermediate mesoderm, underlying the proper $\mathrm{NC}$. Recombination experiments with the DLMZ and animal caps, or grafts of the paraxial mesoderm into ventral epidermis yield expression of NC markers [31, 32, 50]. Furthermore, explants of the NC at neurula stages do not retain expression of $\mathrm{NC}$ markers unless co-cultured with paraxial mesoderm, implicating the mesoderm in the maintenance phase of NC progenitors [31, 32]. A recent study, however, suggests the mesoderm is not necessary for 
neural or NC induction in Xenopus. Injection of an N-terminally truncated form of Cerberus (CerS) inhibits Nodal signaling and mesoderm formation, but still yields expression of Sox3 (a definitive neural marker in Xenopus) and Snail2 at neurula stages [52]. Although they did not analyze later stages to see if the NC was maintained, this is an intriguing finding. The mesoderm appears to be dispensable for NC induction in zebrafish as well; embryos in which Nodal is inhibited and mutant embryos deficient in Nodal signaling lack mesoderm and mesoderm-derived signaling, but still express all NC markers analyzed [53].

In the avian system, various inductive tissues have been reported. Recombination experiments between nascent neural tissue and paraxial mesoderm from later stages (somites/lateral mesoderm) can yield NC derivatives [47]. Juxtaposition of neural and non-neural ectoderm via grafting approaches in vivo, or via explant co-cultures in vitro also yields NC formation [47, 54]. Yet, NC cells can also be generated from epiblast explants at gastrula stages in the absence of mesodermal and neural markers, and without the addition of exogenous signaling molecules [14, $30,55]$. Thus, while the participation of mesoderm seems to be species-specific, it appears that ectodermal signaling alone may be sufficient to trigger $\mathrm{NC}$ induction in the species tested so far. Importantly, there have been no studies regarding the tissue interactions that generate $\mathrm{NC}$ in the mouse or any other mammal.

\section{Major signaling pathways involved in neural crest induction}

The molecular era of NC induction was launched in 1993 with studies identifying important effects of FGF2 and Notch in Xenopus [56, 57], and Dorsalin1 (a TGF- $\beta$ molecule) in chick [58]. A series of studies in the following years firmly established the participation of FGF, BMP, and Wnt signaling [41-44, 59-66]. Today, much progress has been gained in understanding the molecular underpinnings of these and other molecules during the earliest stages of NC formation across different species. Here, we summarize current perspectives on the participation of BMP, Wnt, FGF, and Notch signaling pathways in NC induction among the major model organisms: Xenopus, chick, zebrafish, and mouse.

Bone morphogenetic protein signaling

Bone Morphogenetic Proteins (BMPs) are members of the TGF- $\beta$ superfamily of secreted signaling proteins. BMPs bind to Type I and Type II BMP receptors, and in the canonical pathway lead to activation of Smad1/5/8 proteins. Upon ligand binding, Type I and II receptors form hetero-tetramers, with Type I phosphorylating Smad proteins on their C-terminal domains. Activated Smad1/5/8 proteins then form dimers with $\mathrm{Smad} 4$ and translocate to the nucleus and initiate changes in gene expression. BMP receptors are also capable of activating signaling through other, non-canonical pathways such as those mediated by TAK1 (a MAP kinase kinase kinase), but only the canonical Smad1/5/8 pathway is currently known to act in NC induction. Throughout development, BMPs have multiple roles in axial patterning, cell-fate decisions, and left-right asymmetry, and misregulation can lead to cancers (for a review, see [67-69]).

BMP signaling has a crucial role during early development in the establishment of dorsal-ventral polarity and the promotion of epidermal over neural cell fates. The lateral/ ventral expression of Bmp ligands and the dorsal/medial expression of BMP antagonists provide the potential to create a gradient of BMP activity. Since the NC forms at the interface between medial and lateral tissues, it was proposed that an intermediate level of BMP signaling is necessary for $\mathrm{NC}$ induction. Indeed, epidermal, NC, and neural fates can all be induced in explanted Xenopus ectoderm by increasing levels of Noggin, supporting the gradient hypothesis [44, 70]. A similar BMP gradient model was proposed in zebrafish [71], and recent evidence suggests a BMP gradient may initially specify the prospective NC domain at the late blastula stage [72]. Another interesting study suggests BMP patterns the ectoderm from anterior to posterior progressively during gastrulation [73]. Although the BMP antagonists Chordin, Noggin, and Follistatin secreted from the organizer and dorsal mesoderm are crucial to establishing a gradient, they may not be necessary for NC development in all organisms. In Xenopus, Chordin morpholinos targeted to the DLMZ cause a loss of Snail2 expression in conjugates with animal caps [31], but in zebrafish, morpholino knockdown of all three BMP antagonists still yields a small domain of NC [53]. Interestingly, double-homozygous null mouse mutants for Chordin and Noggin actually present increased expression of NC markers Msx2, Ap2, and Sox10 at early stages. Later NC populations are expanded and undergo precocious migration, suggesting that BMP antagonists in the mouse actually may serve to suppress NC development [74]. Thus, BMP activity might be modulated to levels permissible for $\mathrm{NC}$ induction by other mechanisms in addition to or apart from these secreted BMP inhibitors. Other BMP antagonists have been identified, and support already exists for direct regulation of BMP ligands and intracellular regulation of Smad proteins by FGF/MAPK signaling (topics discussed later).

More recently, an alternative explanation to the gradient model has been put forth. During gastrulation, a partial or complete inhibition of BMP signaling is adequate to create 
a "competency zone" to allow other signals (Wnts, FGFs) to specify the NC $[31,53]$. Then, BMP signaling must be activated in the NPB at neurula stages to allow the full complement of NPB and NC markers to be expressed. An intriguing new study using Xenopus and zebrafish embryos has identified a novel nuclear factor, SNW1, which may mediate this shift in BMP signaling, being responsible for a domain of BMP activity in the prospective NPB at late gastrula stages. SNW1 morphants lack a defined NPB and display clear reductions in early NC markers. Targeted overexpression of $b m p 2 b$ in zebrafish can rescue this phenotype and restore snail2 expression, suggesting the role of SNW1 in NC development is based on its regulation of BMP activity [52].

Recent experiments from chick embryos also suggest that BMP signals act in two phases and argue against a gradient of activity. Treatment of prospective NC explants from gastrula-stage embryos with Noggin for the first $10 \mathrm{~h}$ of culture has no effect on their fate, but treatment after $10 \mathrm{~h}$ causes a loss of NC markers and an induction of neural markers. Similarly, treatment of prospective NC explants with Bmp4 after the first $10 \mathrm{~h}$ of culture causes no change and crest markers arise normally, but if Bmp4 is applied from the beginning of culture, the explants become prospective epidermis [30]. Together, these findings suggest that neural and epidermal cell fates require continued BMP inhibition or BMP activation, respectively, while the $\mathrm{NC}$ is generated by an early phase of inhibition and a late phase of activation. These findings are supported in vivoSmad1/5/8 signaling is essentially absent during gastrulation, but becomes progressively activated throughout the NPB and NNE by neurula stages, with a sharp drop in activity at the NPB/NP boundary $[75,76]$. Also, inhibition of Smad signaling causes a loss of NPB markers [76] and experiments on later-stage (stage 10) chick embryos demonstrate BMP signaling is necessary for the expression of NC specifiers as well [65]. Overall, these studies suggest that BMP/Smad signaling can be completely inhibited at gastrula stages to allow NC induction, but must be activated in the NPB upon neurulation to maintain NC progenitors. This activation of $\mathrm{BMP} / \mathrm{Smad}$ signaling at neurula stages in the NPB may function to promote the NC fate over the neural fate, as the addition of Bmp4 to NP explants from multiple stages can elicit the expression of Pax7, Snail2, and Sox9 [55, 59, 60, 66, 77, 78].

Although the participation of specific BMP ligands has not been directly addressed in the chick, Bmp4 expression closely matches the pattern of Smad1/5/8 activation and is postulated to establish the majority of BMP activity at early stages [75]. Expression of Bmp4 and Bmp7 becomes enriched in the neural folds and adjacent ectoderm at later stages and exogenous Bmp4 protein can induce $\mathrm{NC}$ markers in various contexts in both chick and Xenopus tissues $[30,31,55,59,60,66,77,78]$. In zebrafish, mutants for both bmp $2 b$ (swirl) and bmp7 (snailhouse) lack NC cells, suggesting a shared role in establishing the necessary domain of BMP activity [71, 79]. Notably, bmp2b in zebrafish is proposed to be functionally equivalent to Xenopus Bmp4 [80].

In the mouse, knockouts for Bmp4, Type I BMP receptors $A l k 2$ and $A l k 3$, and Type II Bmpr 2 die before or shortly after gastrulation, precluding analysis of $\mathrm{NC}$ induction [81-85]. However, heterozygous mutants for Bmp4 show some craniofacial abnormalities, suggestive of a role in NC development [86]. Bmp2-null mutants can survive until E10.5 and lack migrating streams of cranial NC cells and do not develop the first two branchial arches [87, 88]. A follow-up study demonstrates Bmp2 is necessary for migration, but not for induction, since Bmp2-null mice do express early NC markers Ap2, Snail1, and Id2 [89]. An epiblast-specific (Mox2-Cre driven) knockout for Alk3 (Bmprla) has been generated, and presents expanded anterior neural markers at the expense of surface ectoderm and caudal neural markers. NC markers Msx1, Pax3, and Sox10 are still expressed, however, suggesting Alk3 is not necessary for initial NC induction in mouse [90]. Another study uses a Pax3-Cre to conditionally remove Alk3 in the prospective NC and demonstrates that early NC markers are still induced in the cranial region, but $\mathrm{NC}$ development in caudal regions is delayed or impaired [91].

A murine line expressing the Cre recombinase from the Wnt1 locus has provided a fruitful tool to analyze later, post-induction events in neural crest development. Wntl is first expressed in the dorsal neural tube, specifically in the $\mathrm{NC}$ population at the midbrain region at the four-somite stage shortly before NC migration. Given the earlier expression of $M s x 1 / 2, \operatorname{Pax} 3 / 7$, and Ap2, Wnt1-Cre lines cannot address events leading to the initial induction of $\mathrm{NC}$ cells, but are valuable for the analysis of later events. A knockdown of Alk2, Alk3, or Alk5 using the Wnt1-Cre causes severe craniofacial, pharyngeal, and cardiac defects, indicating a role for BMP signaling in later NC development [92-95]. Targeted disruption of Smad4 similarly causes multiple craniofacial, pharyngeal, and cardiac anomalies, partially owing to increased levels of apoptosis [96-98]. Although the expression of Msx1/2, Ap2a, Pax3, and $\operatorname{Sox} 9$ at E8.5 is normal in these embryos, expression from E9.5 on is strongly downregulated, implicating Smad signaling in the maintenance of NC markers [98]. Smad4 also participates in Smad2/3 signaling downstream of other TGF- $\beta$ family members, however, so these phenotypes result from a loss of all Smad signaling. Indeed, Wnt1-Cre mediated $T g f b r 2$ knockouts present some of the same defects as those seen in BMP receptor and Smad4 knockouts [99, 100]. Together, these results suggest a later role for BMP/Smad signaling in the mouse, and complement 
the current models from Xenopus and chick that propose a two-phase BMP requirement for NC induction.

\section{Wnt signaling}

Wnts are secreted proteins that initiate a complex cascade of intracellular events, leading to the stabilization of $\beta$-catenin in the canonical pathway. Normally, $\beta$-catenin is phosphorylated by a complex of proteins including GSK3, APC, and Axin, and then subsequently degraded. Upon binding to cell surface receptors belonging to the Frizzled and LRP families, Wnts cause the activation of Disheveled proteins, which inhibit the $\beta$-catenin destruction complex. $\beta$-catenin is then free to enter the nucleus where it associates with TCF/LEF family transcription factors to modulate gene expression. Wnt signaling can also activate non-canonical pathways and promote cytoskeletal changes (via Rho-associated kinase in the PCP pathway) or changes in intracellular calcium levels (through activation of PLC and DAG/IP3 signaling). Wnt signaling is implicated in nearly every facet of development and has roles in the generation of multiple organ systems in the embryo. Wnt signaling also maintains a number of adult tissues, and defects in this pathway commonly contribute to cancers (for a review, see [101, 102]).

Wnt signaling has long been associated with NC induction and has recently been proposed to be the inductive signal. In Xenopus, overexpression of several different Wnt ligands can induce ectopic NPB and NC marker expression, and Wnts are capable of inducing NC markers in conjugation and animal cap assays, but only when combined with BMP antagonists (Chordin/Noggin) [3, 4, 11, 49, 62-64, 103]. Inhibition of Wnt signaling in Xenopus and chick embryos using a variety of extracellular and intracellular modulators has proven the requirement of the canonical $\beta$-cateninmediated pathway for $\mathrm{NC}$ induction and later development [3-5, 7, 9, 10, 14, 31, 49, 63, 64, 66, 78, 104-112].

Wnt3a and Wnt8 have emerged as strong canonical candidates to induce the $\mathrm{NC}$ in Xenopus, being expressed in the DLMZ of the gastrula and at later stages in the caudal $\mathrm{NP}$ and paraxial mesoderm, respectively [7, 31, 49]. Specific knockdown of Wnt3a or Wnt8 using morpholinos or a dominant-negative Wnt8 construct inhibits a panel of NPB and NC markers [4, 7, 31, 49]. Interestingly, Wnt3a morphants still express Wnt8, suggesting Wnt3a may act downstream or independently of Wnt8 in NC induction [7]. Wnt8 is also known to be required for zebrafish NC induction. Zebrafish wnt8 is a bicistronic gene, yielding two transcripts (wnt8.1 and wnt8.2), but only morpholinos that interfere with the translation of Wnt8.1 cause a loss of NC markers pax3, foxD3, and sox10 [113].

In the chick, Wnt $3 a$ and $W n t 8 a / c$ are expressed in the lateral epiblast during blastula stages [114, 115]. At gastrula stages, Wnt $3 a$ is found in the epiblast and primitive streak along with a number of other Wnt ligands (Wntl, $2 b, 7 b$ ), while $W n t 8 a / c$ is expressed in the primitive streak and early mesoderm (S. Chapman, personal communication; http://geisha.arizona.edu/geisha). Addition of Wnt3a to prospective neural epiblast explants can eliminate the expression of neural markers and induce the expression of Msx1, Snail2, and HNK-1 (a marker of migratory NC) [30, 114]. Furthermore, inhibition of Wnt signaling in prospective NC explants causes a loss of NC markers, indicating a requirement for ectodermal Wnt signaling in the chick [30]. Although the potential roles of specific Wnts ligands have not been functionally challenged at early stages, Wnt6 has been implicated in later avian NC development. One study suggests signaling from the NNE activates the canonical pathway in the forming neural folds [66] while another proposes Wnt6 induces the NC through the Rho/JNK non-canonical pathway [116]. Much more work is necessary to determine the source and action of the inductive Wnt molecule(s) in avians, particularly at early stages.

In the mouse, Wntl and Wnt3a are expressed just before $\mathrm{NC}$ migration and participate in later NC development, but do not play a role in the initial induction. Double-homozygous null mutant mice for Wnt1/Wnt3a initially express Ap2 normally, but expression is lost from the migrating cells. Accordingly, these double-mutant mice have severe abnormalities in NC derivatives [117]. A neural-crest specific (Wnt1-Cre) deletion of $\beta$-catenin replicates the midbrain/hindbrain defects of Wnt1 deletion, suggesting Wnt1 signals through the canonical pathway, and additionally presents a near-complete loss of craniofacial structures [118]. Although this $\beta$-catenin null mutant makes up for potential canonical Wnt ligand redundancy, the phenotypes may also be the result of compromised cell adhesion. Studies of other Wnt ligands during early murine development do not reveal obvious NC induction defects (summarized, http://www.stanford.edu/group/nusselab/cgibin/wnt/mouse).

Additional studies have investigated other components of the Wnt signaling pathway. In Xenopus, the Wnt receptor Frizzled7 has been implicated in mediating the initial Wnt signal in the prospective NC domain, with Frizzled3 likely acting at later stages, perhaps responding to Wnt1 signaling [104, 105]. Lrp6, an LDL-receptor related protein, is thought to be a co-receptor for Wnts in $\mathrm{NC}$ induction [109], and participates in signaling with a transmembrane protein, Kremen2 [106]. A novel intracellular PDZ domain-containing protein, Kermit, was also shown to be required for NC development, preferentially mediating Frizzled3 signal transduction [119]. Also in Xenopus, morpholino knockdown of Disheveled 1 or 2 caused an inhibition of Snail2 and Twist, but depletion of 
Disheveled 3 had no effect [120]. A recent study using zebrafish and Xenopus embryos has identified a novel negative regulator of the canonical Wnt signaling pathway, Kctd15; overexpression of Kctd15 inhibits NC markers, while morpholino depletion causes their expansion. A knockdown of Kctd15 rescues Wnt8.1 morphant zebrafish, suggesting Kctd15 acts downstream of ligand binding to decrease signal strength [121]. Another novel regulator of Wnt signaling is ADAM13, a metalloprotease. ADAM13 was shown to be necessary for cranial NC induction, as morpholinos inhibit expression of Snail2, Sox9, and Twist in Xenopus tropicalis embryos. Here, ADAM13 cleavage of Ephrins B1 and B2 is thought to promote Wnt signaling by preventing inhibitory effects of forward EphrinB signaling, thus allowing sufficient Wnt signaling to activate Snail2 and induce the NC [122]. Non-canonical Wnt signaling may be required for NC induction in Xenopus as well - a recent study suggests a crucial role for Wnt11R signaling from the neuroectoderm. The authors demonstrate Wnt11 is capable of activating PAR-1 (also known as microtubule-associated regulatory kinase-MARK) and both molecules are required for early NC markers (Pax3, FoxD3, Sox8), independent of the $\beta$-catenin pathway [123].

Interestingly, a recent study using chick explants proposes Wnt signaling mediates the temporal activation of BMP signaling necessary during the second step of $\mathrm{NC}$ induction. Treatment of prospective neural explants with Wnt3a at gastrula stages upregulates Bmp4 expression and can induce NC markers, while treatment of prospective NC explants with a Wnt inhibitor causes a loss of NC markers and a downregulation of Bmp4 levels [30]. If this same regulation is also present in Xenopus, it would explain why animal caps treated with BMP antagonists and Wnt ligands undergo NC induction: BMP antagonists and Wnt ligands promote the early induction of NPB markers, and then the Wnt ligands activate BMP signaling, overpowering BMP antagonism and leading to the expression of $\mathrm{NC}$ specifiers.

\section{Fibroblast growth factor signaling}

Fibroblast growth factors (FGFs) comprise a large family of secreted polypeptides (22 genes in vertebrates) that bind to transmembrane receptor tyrosine kinases called FGF receptors (four genes, FGFR1-4 in vertebrates) with the assistance of extracellular matrix components, notably heparin sulfate proteoglycans. Following ligand binding, the receptors dimerize and transphosphorylate one another and activate one or more intracellular signaling cascades, including those mediated by Erk1/2 (MAPK), PKC, and PLC-gamma. Alternative splicing has been reported in several ligands as well as FGFR1-3, adding to the complexity and specificity of ligand/receptor interactions and downstream signaling. FGFs have been implicated in multiple aspects of early development, including mesoderm and endoderm formation, gastrulation movements, anterior-posterior and dorsal-ventral patterning, and neural induction among others (for a review, see [124, 125]).

Several Xenopus studies have demonstrated the importance of FGF signaling, with a specific focus on Fgf8a as a NC inducer. This spliceform of Fgf8, unlike Fgf8b, has little to no involvement in mesoderm development [126]. $F g f 8 a$ is expressed in the DLMZ at gastrula stages, but becomes restricted to more posterior tissues at later stages of development [49, 50]. An Fgf8a morpholino inhibits expression of Msx1, Pax3, Hairy2, Snail2, Sox8/10, and Ap2 [3, 4, 11, 49], and unlike Wnts, Fgf8 is capable of transiently inducing NC markers in an animal cap assay without additional BMP inhibitors [50]. Interestingly, overexpression of Fgf8 in low doses expands the expression of numerous NPB and NC specifiers, but in higher doses actually inhibits them [3], suggesting a specific threshold of FGF activity is necessary for NC induction. A recent Xenopus study identified a transmembrane protein, Lrig3, which may participate in modulating FGF levels [127]. Lrig3 seems to enhance Wnt signaling, but inhibits Erk1/2 activation and the NC-inducing activity of FGF ligands, potentially though an interaction with FGFR1. Morpholino analysis demonstrates that Lrig3 operates downstream of Pax3 and Zic1 but upstream of NC specifiers Snail2, FoxD3 and Sox9, and could act in the transition from NPB specification to NC specification.

In Xenopus, FGFs are likely to act during gastrulation and recent experiments suggest the role of FGF is indirect, acting on mesodermal tissues to induce Wnt8 expression [49]. The authors show that Fgf8 overexpression is unable to rescue the loss of Snail2 and Sox8 in Wnt8 or $\beta$-catenin morphants, but overexpression of Wnt8 or $\beta$-catenin can rescue NC deficiencies brought about by an Fgf8 morpholino. Furthermore, the combination of Chordin and Fgf8a in an animal cap assay will induce Pax3, Snail2, and Sox8, but addition of a Wnt8 morpholino blocks induction. They also demonstrate that Fgf8a overexpression can expand the domain of Wnt8 expression and that Fgf8 morphants lack Wnt8 expression in the mesoderm at lategastrula stages. A previous study, however, instead suggests that FGF signaling acts directly on the ectoderm; conjugates of DLMZ and animal caps present strong expression of Snail2, FoxD3, and $\operatorname{Sox} 9$, but conjugates of DLMZ with animal caps injected with a dominant-negative FGFR1 do not [50]. A similar experiment using a dominant-negative FGFR4a did not cause a loss of NC markers, suggesting that signaling through FGFR1 is a key modulator of $\mathrm{NC}$ induction in the ectoderm. In support of a potential ectodermal requirement for FGF signaling, conjugates of neural and epidermal tissue express Snail2, but when the neural portion is injected with dominant-negative 
FGFR1, the conjugates no longer express Snail2 [61]. Moreover, Wnt3a has been implicated in the ectodermal expression of Meis3, a factor capable of directly activating transcription of $F g f 3$ and $F g f 8$ in animal cap assays, suggesting FGF may be activated in the ectoderm [7, 9].

A recent study demonstrates an ectodermal requirement for FGF signaling in chick as well [76]. In this work, inhibition of FGF signaling during gastrulation via electroporation of a dominant-negative Fgfr1 or Mkp3 (inhibitor of MAPK signaling) causes a loss of Pax7 and Snail2, but treatment after gastrulation causes no effect. Using epiblast explants and by restricting electroporation to the prospective NPB, this study demonstrates that FGF/ MAPK signaling within the gastrula epiblast is required for $\mathrm{NC}$ induction. Interestingly, FGF receptors 1 and 4 are expressed in the prospective NC epiblast during this time, but are not found in the mesoderm [76, 128], suggesting FGF does not act on the mesoderm in avian NC induction. FGF signaling is necessary for mesoderm formation [129], however, and many FGF ligands are expressed in the mesoderm and primitive streak (http://geisha.arizona.edu/ geisha; 130]). During the stages leading up to gastrulation, Fgf8 is expressed in the hypoblast (the tissue underlying the epiblast), and is thought to participate in neural induction [131], making it an attractive candidate to act on the prospective NC epiblast. Still, the source of the inductive ligand has not yet been identified in the chick, and other FGFs including Fgf3 are expressed in the epiblast itself [132]. The highest level of FGF/MAPK activity during gastrulation is found in the primitive streak [76, 128], and during this and later stages, it is known to regulate the expression of multiple Wnt ligands expressed there, including Wnt3a, Wnt8a/c, and Wnt5b [76, 129, 133]. However, FGF was also found to positively regulate antagonists of the canonical Wnt signaling pathway including NOTUM, Sizzled, Sfrp2, and Cerberus [129]. It would be interesting to determine whether FGF/MAPK regulation of these molecules is necessary for $\mathrm{NC}$ induction in addition to its activity within the prospective $\mathrm{NC}$ epiblast itself.

A requirement for FGF signaling in zebrafish NC induction has not yet been proven, but FGF/MAPK signaling is crucial for dorsoventral patterning during gastrulation and overexpression of Fgf8 causes an expansion of Ap2 [134, 135]. In the mouse, several FGF molecules are known to act early in development, but have not been functionally linked to NC induction. Null mutants for Fgf4 [136] and Fgfr2 [137] display defects in the inner cell mass, and knockouts for Fgf8 [138, 139] and Fgfr1 [140-142] do not gastrulate properly and thus die before $\mathrm{NC}$ formation. Studies have not yet assessed later contributions of these molecules, but all other FGF knockouts generated so far appear to undergo normal NC induction (see [143] for a summary).
In addition to its ability to modulate the Wnt pathway, FGF/MAPK signaling also contributes to BMP antagonism on multiple levels. In Xenopus and zebrafish, FGF signaling positively regulates the expression of Chordin and Noggin during gastrulation [144-146], and negatively regulates BMP ligand expression in the chick and zebrafish $[114,132,135]$. A recent study also shows FGF positively regulates SNW1 in the chick [129], a molecule thought to modulate BMP signaling in Xenopus [52]. Additionally, a compelling intracellular regulation of Smad1 has been uncovered, directly linking MAPK signaling to Smad inhibition [147]. MAPK was found to phosphorylate the linker region of Smad1, leading either to Smurf1-mediated polyubiquitination and degradation or exclusion from the nucleus [147-149]. This pathway was shown to be crucial to Xenopus neural induction [148, 150, 151], and may operate similarly to cell-autonomously regulate Smad activity in the prospective NPB. Even more intriguing, GSK3, active in the absence of canonical Wnt signaling, was also shown to phosphorylate the Smad1 linker, downstream of MAPK phosphorylations [148]. This provides yet another mechanism whereby Wnt signaling could promote BMP signaling. These findings suggest Smad signaling is a platform to integrate signals from the BMP, Wnt, and FGF pathways. If this is found to be conserved across species, it could account for the reported formation of NC in zebrafish lacking dorsal BMP antagonists (Chordin/Noggin/Follistatin) [53].

\section{Notch signaling}

Notch proteins are transmembrane receptors activated by binding to transmembrane ligands on the surface of adjacent cells. Following ligand binding, multiple cleavage events occur, leading to the intracellular release of the Notch intracellular domain (NICD). The NCID then translocates to the nucleus where it converts the recombining binding protein suppressor of hairless complex from transcriptional repressor to an activator with the help of proteins from the mastermind-like protein family. The NCID is also able to participate in additional transcriptional activation processes, independent from this canonical pathway. Notch signaling has been implicated in numerous developmental processes, particularly involved in establishing boundaries between different cell types (see [152] for a review).

Studies from Xenopus demonstrate a crucial role for Notch/Delta signaling, but the precise time of its activity is still uncertain. Glavic et al. show that Notch is expressed in the prospective NC territory, while ligands Delta and Serrate are expressed in the surrounding regions. They propose that Delta1 interacts with Notch to activate the transcription factor Hairy2, which then suppresses Bmp4 
signaling, allowing the inductive phase to proceed [8]. However, this suppression appears to occur during the second maintenance phase, when BMP signaling must be activated. A more recent study using the same tools reports contradictory results, perhaps due to slight differences in the stage of treatment. This group shows that Hairy2 is not regulated by Notch, but is positively regulated by BMP inhibition, the canonical Wnt pathway, and Fgf8, and is downstream of Msx1, Pax3, and Zic1. They propose that Hairy2 maintains NC progenitors, as overexpression represses NC markers and upregulates NPB markers [11]. A follow-up study finds that Hairy 2 actually activates the Notch pathway cell-autonomously, activating Delta1 via STAT3. Delta1 then acts non-cell-autonomously to upregulate Id3, Snail2, and Sox9 [12]. This suggests Hairy2 may act as a trigger for Notch/Delta signaling at the maintenance phase, eventually leading to the expression of NC specifiers. Another Xenopus study extensively characterizes a novel secreted protein, Tsukushi (Tsk), necessary for NC induction. Tsk is capable of acting as a BMP antagonist through direct binding to Bmp4, while also regulating the Notch pathway by binding to the extracellular domain of Delta1. The authors propose Tsk is essential to establish the proper level of BMP signaling at the prospective NPB during gastrulation, together with Notch signaling [153]. Potentially, Hairy2 and Tsk serve to modulate Notch and BMP signaling at multiple levels of NC development, but more research is needed.

In the chick, one study has assessed Notch signaling, demonstrating a role in refining the $\mathrm{NC}$ domain after induction has taken place. In this instance, Notch seems to act indirectly. Both overactivation and inhibition of Notch signaling cause an inhibition of Bmp4 in the epidermis and Snail2 in the neural fold, but overexpression of Bmp4 in these embryos can rescue the loss of Snail2 expression. This suggests Notch acts primarily to regulate Bmp4 levels [154]. Notch has yet to be implicated in NPB specification during gastrulation in chicks.

Notch signaling has also been linked to NC development in zebrafish embryos, though it seems to act primarily by restricting the neural domain. A loss of Notch/Delta signaling in mindbomb (mib) mutant zebrafish causes a loss of $\mathrm{NC}$ derivatives at the expense of lateral NP derivatives such as interneurons [155]. Another study suggests Notch acts via repression of Neurogenin-1 function, restricting neurogenesis without actively promoting $\mathrm{NC}$ formation [156]. More recent studies suggest Notch/Delta acts earlier, refining the border of the neural plate specifically through negative regulation of the transcription factor prdmla (Blimp1) [157]. Prdm1a, necessary for NPB specification in zebrafish, antagonizes another factor olig4, which defines the lateral edge of the NP and promotes neural cell fates over NC [23, 157, 158]. It appears olig4 is restricted by
BMP signals during gastrulation, as swirl/bmp $2 b$ mutant zebrafish demonstrate a laterally expanded expression of olig4 [158]. Importantly, in all these zebrafish studies, inhibition or loss of Notch signaling primarily affected trunk, but not cranial NC cells, suggesting it is not responsible for the initial induction of all $\mathrm{NC}$ cells.

Mouse mutants for members of the Notch signaling pathway generally display an increase in neuronal differentiation markers and a decrease in progenitor markers, demonstrating a critical role in the early stages of CNS development (for a review, see [159]), but there is no support for a role in $\mathrm{NC}$ induction. Homozygous null mutants for Delta1 display proper generation of NC cells, but show defects in migration and differentiation [160], consistent with a later role for Notch signaling.

\section{Other signaling pathways}

Retinoic acid (RA) signaling has an established role in caudal neural patterning, and may act in NC development as well, though it seems to act after the initial induction. RA signaling is restricted to the caudal portions of the embryo during early development, owing to the posterior localization of the RA-synthesizing enzymes (retinaldehyde dehydrogenases, Raldhs) and the anterior localization of RA-degrading enzymes (Cyp26 family members). A study using Xenopus animal caps showed that induction of Pax3 by chick mesoderm or NP does not require RA [161] suggesting RA is not necessary for NC induction. Another study using Xenopus embryos demonstrated that addition of exogenous RA or over-activation of RA signaling can expand Snail2 expression anteriorly, whereas treatment with a dominant-negative RA receptor causes a posterior expansion [110], suggesting RA effects on the NC are secondary to axial patterning. An avian study using vitamin A-deficient (VAD) quails (which lack the RA precursor) suggests RA is required for the survival of migrating NC cells; VAD quails appear to form cranial NC cells properly, but within a few hours of migration, they undergo extensive apoptosis [162]. A mouse study evaluated RA signaling in the cranial neural crest and provides a slightly different perspective [163]. As a result of knocking out both Cyp26a1 and c1 together, RA signaling is expanded anteriorly, and although NC markers Snail and Sox9 are expressed normally in the cranial neural folds, migrating $\mathrm{NC}$ cells are largely absent. Interestingly, crossing Cy26a1/ c1 double mutants with a null mutant for Raldh2 (the only RA-synthesizing enzyme present at these stages) rescues the NC migration defect, despite the expected absence of RA signaling in these embryos. This suggests that although over-activation of RA signaling can disrupt cranial NC migration, endogenous RA signaling is not required for migration. Furthermore, because NC markers were initially 
expressed normally, RA signaling does not appear to function early in NC induction in the mouse.

A recent study evaluates the role of Indian Hedgehog (Ihh) in early neural crest development in Xenopus [164]. Here, the authors demonstrate Ihh signaling is necessary for NC induction, maintenance of progenitors; loss of Ihh function using morpholinos, dominant-negative constructs, and chemical inhibition causes a loss of both NPB and NC specifiers and an expansion of neural and epidermal markers. They reveal requirements for autocrine signaling within the prospective $\mathrm{NC}$ and paracrine signaling from the mesoderm, with mesoderm-based signaling further being necessary for proper migration. A well-characterized member of the hedgehog family, Sonic hedgehog (Shh), has established roles in the formation of left/right asymmetry and ventral patterning of the spinal cord, and although ectopic application of Shh can repress NC markers at later stages in the chick [59, 65, 165], an endogenous role in NC induction has yet to be proven.

Lastly, Endothelin signaling has also been implicated in Xenopus NC induction [32]. Using a combination of in vivo and explant approaches, the authors suggest Endothelin-1, released from the mesoderm, functions in both NC specification and cell survival. They demonstrate that morpholino knockdown of the Endothelin-A receptor and chemical inhibition of Endothelin signaling causes a loss of NC specifiers FoxD3, Sox9 and Sox10, but not the NPB specifier Msxl, suggesting a role in NC progenitor maintenance during the mid-neurula stage. With $\mathrm{NC}$ induction requiring the precise temporal regulation of multiple transcription factors, additional signaling pathways are surely yet to be uncovered.

\section{Perspectives}

Posteriorization and early inducers

Wnts, FGFs, and retinoic acid (RA) are proposed to act as caudalizing or posteriorizing factors during early development. In neural development, it is thought that the NP is initially composed entirely of rostral or anterior character, subsequently being posteriorized by the action of Wnts, FGFs, and RA to give rise to caudal components of the nervous system (reviewed in $[166,167]$ ). Since these same factors are involved in $\mathrm{NC}$ induction, it was proposed that the NC is a result of NPB posteriorization [110]. A study in chick, however, indicates the prospective NPB is initially specified by gastrula stages to become $\mathrm{NC}$ at all axial levels, requiring the inhibition of Wnt signals at later stages to allow placodal development anteriorly [30]. Additionally, work in Xenopus suggests Wnt signaling acts directly to induce the $\mathrm{NC}$, independent of its role in antero- posterior neural patterning [111]. Recent Xenopus studies identify two factors, Gbx2 and Meis3, as direct targets of $\mathrm{Wnt} / \beta$-catenin signaling necessary for $\mathrm{NC}$ induction $[7,10]$. Although both of these genes are involved in caudal neural patterning, the NC-inducing activity of Gbx2 is separable from its role in NP posteriorization [10]. Gbx2 is also shown to cooperate with Zic1 to specify the NC fate, while Zic1 activity alone leads to placodal development $[3,10]$. These findings argue that NPB antero-posterior patterning and $\mathrm{NC}$ induction are distinct from events in early neural development.

Although these studies describe Gbx2 and Meis 3 as some of the earliest-expressed proteins necessary for NC induction and direct targets of Wnt signaling, another recent study proposes Ap2a is the earliest-acting factor in Xenopus [6]. Ap2a is broadly expressed, including the prospective NPB from the onset of gastrulation (stage 10) and preceding the expression of all other NPB specifiers but still downstream of $\mathrm{Wnt} / \beta$-catenin signaling. They demonstrate that morpholino depletion of Ap2a causes a loss of Msx1, Hairy2, Pax3, and Snail2, and a gain-offunction upregulates them. Although the initial expression of Ap2a is not affected by morpholinos against other NPB specifiers, later expression is. A zebrafish study also suggests Ap2a (Tfap2a) is a critical early regulator of the NC fate, along with FoxD3 [21]. These two factors are expressed during gastrulation and are necessary and sufficient for NC development. Interestingly, double-mutants of Ap2a and FoxD3 actually present altered patterns of BMP and Wnt signaling, suggesting these factors participate in establishing the signaling environment required for $\mathrm{NC}$ induction. The expression patterns of Ap2 and FoxD3 in the chick, however, are not consistent with an early role in $\mathrm{NC}$ induction [16]. Instead, Pax7 is currently the earliestexpressed factor necessary for $\mathrm{NC}$ induction in the chick [14]. This marker is expressed exclusively in the NPB soon after gastrulation and later labels the entire neural folds. This contrasts with other NPB specifiers that are found more lateral (Msx1, Pax3) and NC specifiers, which are initially only expressed in the cranial NC (Snail2, FoxD3, Sox9) [16].

Neural and neural crest induction: shared first steps?

Recent studies in the chick and frog describe the initial induction of the $\mathrm{NC}$ taking place during gastrulation, requiring the activation of FGF and Wnt signaling and the inhibition of BMP signaling. Studies from these same organisms suggest neural induction also requires BMP inhibition and the activation of FGF/Erk signaling from the blastula to the gastrula stage $[114,131,132,168-170]$ (reviewed by [171]), prompting the question of whether neural and NC cells are initially specified together. 
In the chick, Erk1/2 proteins are activated throughout the epiblast, encompassing both the prospective NP and prospective NPB from blastula to gastrula stages [76, 128]. Although studies of $\mathrm{NC}$ induction have not analyzed effects of FGF/Erk inhibition at pre-gastrula stages, inhibition at gastrula stages causes a loss of NPB markers in their endogenous domain, but an upregulation in the NP [76]. Thus, if FGF is to act similarly on these populations, it likely does so before gastrulation. Furthermore, activation or inhibition of Wnt signaling can interchange the expression of neural and $\mathrm{NC}$ markers in explants taken at pre-gastrula [30, 114] but not post-gastrula stages [55], suggesting Wnts mediate the initial choice between these fates before gastrulation. Indeed, Wnt ligand expression and nuclear $\beta$-catenin are found in the lateral epiblast, but absent from the medial, neural-specified epiblast at blastula stages [114, 115, 172]. Furthermore, in Xenopus, inhibition of Wnt signaling causes a strong expansion of neural plate markers at the expense of NC and placodal markers. Yet, upregulation of canonical Wnt signaling interferes with neural induction, and this activity can be separated from Wnt-mediated NC induction [107].

In Xenopus animal cap assays, addition of BMP antagonists such as Chordin or Noggin is sufficient to induce neural markers (the animal caps are said to be "neuralized"). To launch and maintain NC markers in this assay, caps must first be neuralized and then treated with FGF or Wnt agonists, supporting a potential shared requirement for BMP antagonism. However, BMP antagonism seems to behave differently toward markers of neural and $\mathrm{NC}$; inhibition of BMP signaling can induce ectopic neural and $\mathrm{NC}$ markers prior to the blastula stage, but inhibition at early gastrula stages only generates ectopic NC [173]. Similarly, inhibition of BMP signaling in lateral ectoderm of the chick at gastrula stages can induce ectopic NPB and NC markers [174], but not neural markers [169]. Interestingly, BMP ligands are expressed throughout the epiblast at blastula stages [132, 175], despite the seeming requirement for BMP inhibition to initially promote both neural and NC fates. Perhaps signaling at the blastula stage in chick is primarily utilized to maintain the pluripotency of the epiblast, similar to the situation in the mouse (discussed below).

Early signaling insights from mouse studies

Although the BMP, Wnt, and FGF signaling pathways are needed to establish cell fates at gastrula and neurula stages, studies of mouse embryonic stem (ES) cells (derived from the inner cell mass of the blastocyst) and epiblast stem cells (EpiSCs) suggest dynamic and temporally segregated roles earlier in development. The FGF/Erk signaling cascade must be inhibited to allow self-renewal and pluripotency in mouse ES cells, with activation of the pathway driving them towards differentiation (summarized in [176]). Along with Erk inhibition, GSK inhibitors are required to retain a pluripotent state, but this activity seems to be independent of the Wnt pathway. Alternatively, leukemia inhibitory factor (LIF) and Bmp4 are capable of maintaining mouse ES cells in an undifferentiated state. Autocrine Fgf4/Erk signaling appears to push mouse ES cells towards differentiation [177] and Erk signaling seems to be necessary for their adoption of the neural fate [170], but an in vivo study of mouse development suggests FGF signaling is not necessary for neural induction [178]. Studies of earlier development support an endogenous role for BMP signaling [178] and FGF/Erk inhibition [179] in maintaining pluripotency of intact embryos, suggesting the initial differentiation activity of FGF may be sufficient to establish the competence of the epiblast for neural development. In support, the addition of exogenous FGF ligands is essential for the self-renewal and pluripotency of EpiSCs (derived from the gastrula epiblast), with inhibition of the FGF/Erk signaling leading to differentiation [180]. This is in stark contrast to mouse ES cells where FGF/Erk signaling provides the opposite instructions. Indeed, FGF signaling in EpiSCs actually serves to inhibit neural induction [181]. Together with ES cell studies, these findings suggest FGF has an initial role in epiblast formation, but then serves to maintain pluripotency of the epiblast [182]. These changing roles of FGF signaling caution us to consider temporal differences in cellular responses and underscore the dynamic nature of signaling pathways.

\section{Human neural crest development}

Advancing our limited understanding of human NC development will surely improve our capacity to address the many human maladies associated with improper NC development. Model organisms have provided invaluable information on $\mathrm{NC}$ development and while many molecules and processes are conserved (as described above), the deviations that are present make it imperative to specifically study human NC biology. The morphology of early human NC development has been depicted from careful histological analysis [183]. An extensive molecular profiling study was performed on cell lines derived from human neural tube explants, presumed to be $\mathrm{NC}$, and indicates the human NC shares many markers with stem cells. Additionally, the study suggests conservation among $\mathrm{NC}$ cells of the chick and mouse, but also points to unique traits in the human NC [184]. More recently, a battery of NC markers were analyzed in cranial and trunk regions of intact early human embryos (Carnegie stages 12-18), confirming a broad conservation of expression profiles with model organisms [185]. This study identified Pax3, Sox9, 


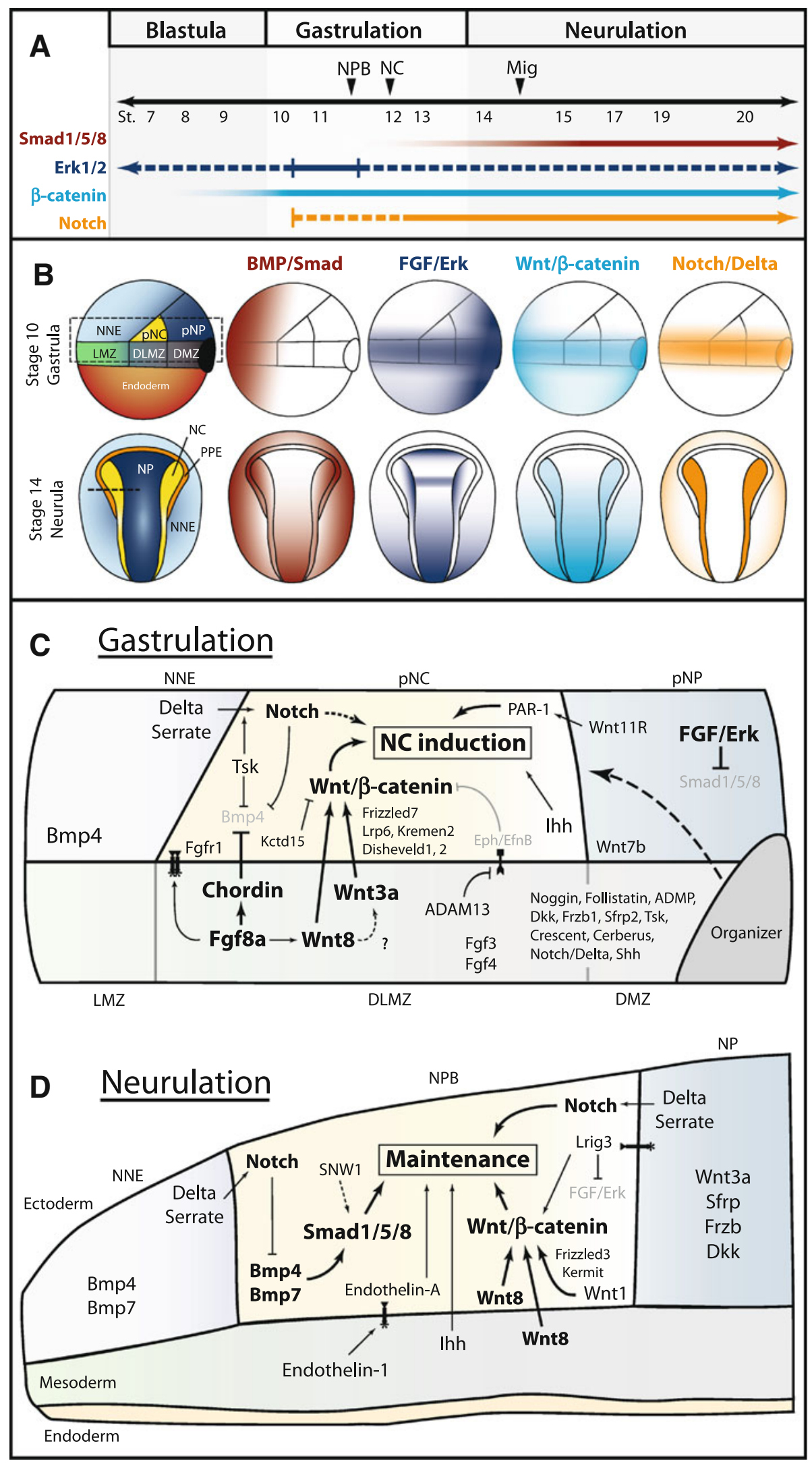


4 Fig. 3 Temporal and spatial participation of signaling molecules involved in Xenopus neural crest induction. a Timeline of signaling pathway activation and requirement in early $\mathrm{NC}$ development. Closed arrows/lines indicate activation and requirement in $\mathrm{NC}$ tissues. Dotted lines indicate activation in NC tissues, but requirement is unknown. BMP/Smad signaling must be inhibited during gastrulation, but activated upon neurulation. The specific stage when Smad signaling first becomes activated in the $\mathrm{NC}$ has not been determined. FGF/Erk signaling is activated throughout early NC development, but has only been functionally implicated during early gastrula stages [49, $50]$. Wnt $/ \beta$-catenin signaling is thought to be required at all stages of early NC development, but becomes more strongly activated by neurulation [31]. The precise time when Notch is required is still debated, but may play a role in the initial induction during gastrulation. b Spatial activation of BMP, FGF, Wnt, and Notch signaling during Xenopus gastrulation and neurulation. BMP/Smad, FGF/Erk, and Wnt/ $\beta$-catenin activation based on data from [199, 200]. Notch/Delta activation inferred from requirements in germ layer segregation and NC development $[8,12,201]$. Overall, spatiotemporal activation of these pathways is conserved between Xenopus and zebrafish. c Spatial expression and participation of signaling molecules in Xenopus neural crest induction at the gastrula stage. Diagram corresponds to dotted box of stage 10 gastrula in b. Molecules in bold have support from multiple studies. Solid lines indicate known relationships, dotted lines indicate potential relationships. NC induction results from the combined action of $\mathrm{Wnt} / \beta$-catenin, FGF, Indian Hedgehog, and non-canonical Wnt signaling. Fgf8a is thought to regulate the expression of Wnt8 in the dorsolateral marginal zone (DLMZ), but may signal to the prospective neural crest itself. Wnt8 and Wnt3a signaling from the DLMZ activate canonical Wnt signaling in the prospective neural crest. Multiple agonists and antagonists of BMP and Wnt signaling are expressed in the Organizer, DMZ, and DLMZ and function in dorsal-ventral and anteriorposterior patterning, and these molecules likely also participate in NC induction (dotted line). Expression of other potential signaling molecules and regulators is presented. See main text for details on the participation of individual signaling molecules. $\mathbf{d}$ Participation of signaling molecules in the maintenance of NC progenitors in Xenopus neurulation. Diagram corresponds to section at dotted line in stage 14 neurula in b. NC maintenance requires activation of Smad1/5/8, Wnt/ $\beta$-catenin, Notch/Delta, Indian Hedgehog, and Endothelin-A signaling. BMP and Wnt signals are likely mediated by Bmp4, Bmp7, Wnt1, and Wnt8, expressed in the neural folds upon neurulation. Additionally, Wnt8 is present in the paraxial mesoderm and Wnt3a in the neural plate. Notch signaling is thought to operate both by regulating Bmp ligand levels and leading to the expression of NC specifiers. See main text for details on the participation of individual signaling molecules. Expression data gathered from references in text and from http://www.xenbase.org

and Sox10 expression in pre-migratory $\mathrm{NC}$ at early stages of caudal trunk development with Ap2, Pax7, Sox9, and Sox 10 expressed during early migration at more anterior locations. Of note, HNK-1 identified few migratory NC and while p75 recognized many more, it only labeled a subset of NC cells. More importantly, p75 also labeled many non$\mathrm{NC}$ cells. This is particularly relevant, given the broad use of these two markers amongst stem cell biologists (see below).

Given the obvious experimental limitations and restrictions of studying early human development, human embryonic stem cells (hESCs) provide an excellent alternative to study the human NC. While it is difficult to argue how close this system replicates embryonic development, it does have the capability to challenge the potential of cells in many different contexts and to expose used mechanisms and restrictions. hESCs can be induced to form NC-like cells capable of differentiating into nearly all known NC derivatives. These progenitor cells have been generated by several different protocols in varying culture conditions [186-198]. Initial work relied on stromal cell co-cultures [186, 188, 191], and later progressed to derivation from embryoid bodies [187, 198] or neurospheres [192, 193, 196]. Cleaner and more efficient protocols have been recently reported to derive NC from hESCs in cultures of adherent cells in serum-free defined media, without complex intermediary structures [190, 194, 197].

Culture density of hESC was reported to alter neural and NC formation in a differentiation protocol including FGF2, Insulin, and gradual exposure to both BMP and Nodal/ Activin inhibition (Noggin and SB431542) [190, 194]. Another study demonstrated that both BMP and Wnt signaling were necessary for $\mathrm{NC}$ markers in cells derived from neural rosettes, but it was unclear whether these signals were required for the initial marker expression, or for their maintenance and the generation of derivatives [193]. In several of these pioneering studies, HNK-1 and p75 were used to screen for NC progenitors, but recent findings highlight complications in the use of these markers. In the human, p75 marks only subsets of NC and additionally labels many non-NC cells, while HNK-1 labels a smaller fraction of NC [185], and in at least one study, their use was shown ineffective to discriminate between $\mathrm{NC}$ and non-NC [193]. Still, HNK-1-positive, p75-positive cells induced from hESCs do exhibit the capacity to generate an array of derivatives characteristic of the NC. In some of these studies, NC-like cells arise from Pax6+ neural precursors, while in others, an alternative origin has been proposed. Yet, no studies have addressed their possible equivalence or distinct differentiation potential. An intriguing recent study reports a direct protocol for the generation of NC progenitors from hESCs in 12-14 days using a GSK3- $\beta$ inhibitor (BIO) to activate canonical Wnt signaling, Noggin to inhibit Smad1/5/8 signaling, and SB431542 to inhibit Smad2/3 signaling [197]. Interestingly, removal of Noggin has no effect, perhaps owing to the low level of basal Smad1/5/8 activation, but addition of BMP4 suppresses the generation of these cells, suggesting high levels of BMP/Smad signaling are not conducive to the formation of NC-like cells from hESCs. These conditions mimic some of those currently thought to induce the $\mathrm{NC}$ in the model organisms studied, and underscore the value of continued research in NC induction. Reciprocally, the study of hESC biology will undoubtedly unveil exciting new insights into the signaling events in NC development in vivo. 


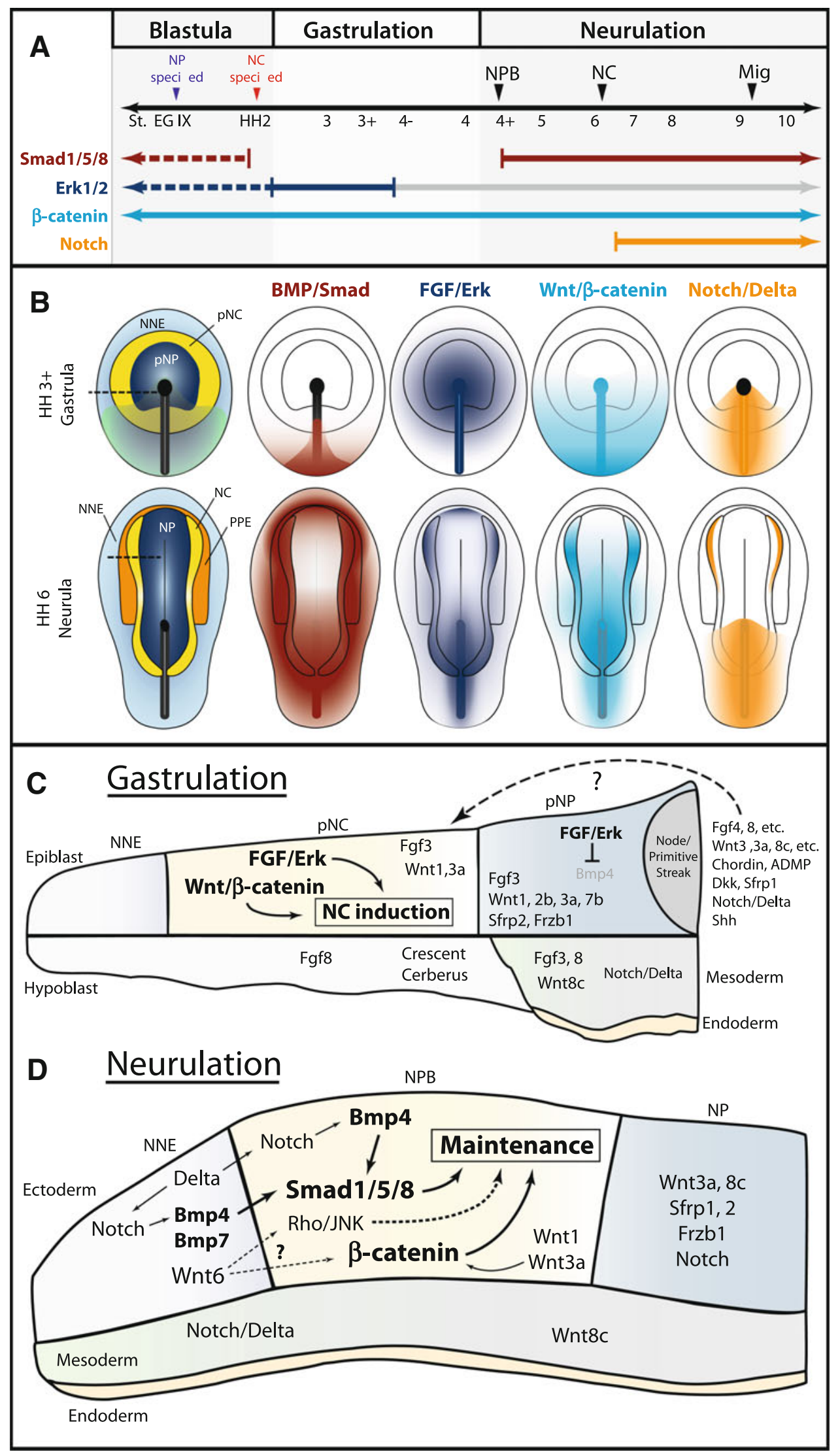


४Fig. 4 Temporal and spatial participation of signaling pathways involved in chick neural crest induction. a Timeline of signaling pathway activation and requirement in early $\mathrm{NC}$ development. Closed arrows/lines indicate activation and requirement in $\mathrm{NC}$ tissues. Dotted lines indicate activation in NC tissues, but requirement is unknown. Smad1/5/8 signaling is active in the entire epiblast at blastula stages, but is inactivated by gastrulation [75]. Signaling becomes active with the expression of NPB markers, and remains active through to migration. Erk signaling is also active in most of the epiblast at blastula stages, and is required for neural induction until gastrulation. A requirement for FGF/Erk signaling in NC induction was only demonstrated during gastrulation. Erk signaling remains active in the NPB and NC tissues through to migration, but is no longer required for $\mathrm{NC}$ development (gray line). Wnt $/ \beta$-catenin signaling is thought to be necessary for all stages of early $\mathrm{NC}$ development. A requirement for Notch/Delta signaling was demonstrated at mid-neurula stages. b Spatial activation of BMP, FGF, Wnt, and Notch signaling during chick gastrulation and neurulation. BMP/ Smad activation based on [76]. FGF/Erk activation based on [76, 128]. Wnt/ $\beta$-catenin activation inferred from expression of agonists and antagonists, and functional requirements for Wnt signaling. Notch/Delta activation is based on expression of molecules and functional requirements [154]. c Spatial expression of relevant signaling molecules and requirements for chick neural crest induction during gastrulation. Diagram corresponds to section at dotted line $\mathrm{HH}$ $3+$ gastrula in b. Functional studies have demonstrated a requirement for FGF/Erk and $\mathrm{Wnt} / \beta$-catenin signaling, but the participation of specific signaling molecules has not been challenged. The spatial expression of some potential signaling molecules is presented. Multiple FGF and Wnt agonists and BMP and Wnt antagonists are expressed in the node/primitive streak, but it is unclear whether these molecules can diffuse the distance to influence the prospective NC tissue (dotted arrow). d Spatial expression and participation of signaling molecules and pathways in the maintenance of $\mathrm{NC}$ progenitors during chick neurulation. Diagram corresponds to section at dotted line in HH 6 neurula in b. Smad1/5/8 and $\beta$-catenin are likely activated by Bmp4, Bmp7, Wnt1, and Wnt3a expressed in the neural folds and adjacent NNE. Wnt6 in the NNE has also been implicated in NC development, but may act through the noncanonical Rho/JNK pathway. Notch signaling likely participates indirectly by regulating Bmp4 expression. Spatial expression of other potential signaling molecules is presented. Expression data gathered from references in the text and from http://geisha.arizona.edu/geisha

\section{Summary of inductive signals}

As we learn more about the molecular events leading to the specification of the neural crest, we unveil subtleties in the induction mechanism employed by each organism. Graphical summaries of the spatiotemporal activation of signaling pathways and participation of signaling molecules are presented for the two most extensively studied organisms, Xenopus (Fig. 3) and chick (Fig. 4). Taking the information from these two models and integrating findings from zebrafish and mouse, an overall theme of neural crest induction has emerged. The initial induction of the neural plate border appears to involve signaling events from the blastula to the gastrula stage, with continued signaling taking place during neurulation to maintain neural crest progenitors and bring about the expression of neural crest specifiers. In this model, FGF and Wnt signaling are

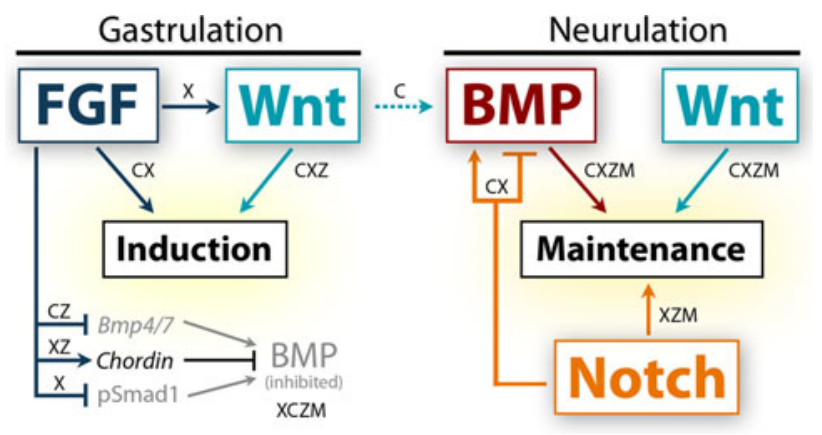

Fig. 5 A model of signaling participation during the two phases of neural crest induction. In this figure, we present a simplified model of the major signaling events thought to occur in NC induction, drawing on evidence from all four of the organisms discussed. Since the precise time, source, and cross-regulation between pathways vary between species, the model organism is noted where a specific interaction or activity is known to occur. $X$ Xenopus, $C$ chick, $Z$ zebrafish, $M$ mouse. During gastrulation, FGF and Wnt signaling are both known to induce the neural crest at the prospective NPB, activating the expression of NPB specifiers. Xenopus studies demonstrate that FGF regulates Wnt signaling during this first phase, but evidence from chick and Xenopus suggests FGF acts directly as well. BMP signaling must be at least partially inhibited for this first step, and FGF participates in BMP attenuation on multiple levels. The transition to the second phase involves the activation of BMP signaling, and research on chick explants suggests Wnts may participate in this BMP activation. Since FGF contributes to BMP antagonism before and during gastrulation, the restriction of FGF activity or insensitivity of the NPB to FGF signals also likely plays a role in this transition. In the second phase, BMP and Wnt signaling converge to maintain the expression of NPB specifiers and initiate the expression of NC specifiers. Notch signaling is known to refine the domain of BMP activity, but some evidence suggests Notch acts directly on the neural crest population as well. Throughout later neural crest development, these signaling pathways continue to participate in migration and differentiation

required for the initial phase of neural crest induction with Wnt, BMP, and Notch signals acting later to maintain neural crest development. A simplified model is presented in Fig. 5.

The first phase requires at least a partial attenuation of the BMP/Smad signaling pathway, but by neurula stages BMP signaling must be activated. BMP inhibition is crucial for both neural and neural plate border specification, and might be regarded as a required step in establishing the competency of the prospective ectoderm. BMP attenuation is likely achieved though multiple redundant methods, including the limited expression of BMP ligands, the activity of secreted BMP antagonists (Chordin, Noggin, and others), and FGF/MAPK-mediated intracellular Smad inhibition. The activation of BMP signaling at neurula stages may be temporally regulated by Wnt signaling. Additionally, the canonical Wnt signaling pathway must be activated within neural crest progenitors themselves and throughout early neural crest development. Analogous to other developmental processes, Wnt signaling is capable of 
mediating cell-fate decisions, and likely directs the choice between neural and neural crest progenitors. Notch also promotes neural crest development over neural cell fates at multiple points in development, but the precise mechanism(s) remain to be understood. Lastly, a functional FGF signaling pathway is necessary for the initial induction of neural plate border specifiers. However, FGFs are capable of regulating Wnt and BMP signaling and are crucial for several other developmental events (including neural induction, mesoderm development, gastrulation movements, and early epiblast pluripotency and competence), all of which could influence NC induction. In the years to come, the real challenge will be to understand the crossregulation and combinatorial inputs of these and other signaling pathways, their precise temporal effects, and how they integrate to establish the neural crest program. Since recent studies have found that the NC is specified at pregastrula stages, a more thorough analysis of these signaling pathways is called for at early stages of development to understand the complete transcriptional cascade of events that enable the amazing plasticity of the neural crest.

Acknowledgments We thank E. Betters for contributing to Fig. 1 and B. Murdoch for comments on this manuscript.

Open Access This article is distributed under the terms of the Creative Commons Attribution License which permits any use, distribution, and reproduction in any medium, provided the original author(s) and the source are credited.

\section{References}

1. Meulemans D, Bronner-Fraser M (2004) Gene-regulatory interactions in neural crest evolution and development. Dev Cell 7(3):291-299

2. Sauka-Spengler T, Bronner-Fraser M (2008) A gene regulatory network orchestrates neural crest formation. Nat Rev Mol Cell Biol 9(7):557-568

3. Hong CS, Saint-Jeannet JP (2007) The activity of Pax3 and Zic1 regulates three distinct cell fates at the neural plate border. Mol Biol Cell 18(6):2192-2202

4. Monsoro-Burq AH, Wang E, Harland R (2005) Msx1 and Pax3 cooperate to mediate FGF8 and WNT signals during Xenopus neural crest induction. Dev Cell 8(2):167-178

5. Sato T, Sasai N, Sasai Y (2005) Neural crest determination by co-activation of Pax3 and Zic1 genes in Xenopus ectoderm. Development 132(10):2355-2363

6. de Croze N, Maczkowiak F, Monsoro-Burq AH (2010) Reiterative AP2a activity controls sequential steps in the neural crest gene regulatory network. Proc Natl Acad Sci USA 108(1): $155-160$

7. Elkouby YM, Elias S, Casey ES, Blythe SA, Tsabar N, Klein PS, Root H, Liu KJ, Frank D (2010) Mesodermal Wnt signaling organizes the neural plate via Meis3. Development 137(9):15311541

8. Glavic A, Silva F, Aybar MJ, Bastidas F, Mayor R (2004) Interplay between Notch signaling and the homeoprotein Xiro1 is required for neural crest induction in Xenopus embryos. Development 131(2):347-359

9. Gutkovich YE, Ofir R, Elkouby YM, Dibner C, Gefen A, Elias S, Frank D (2010) Xenopus Meis3 protein lies at a nexus downstream to Zic1 and Pax3 proteins, regulating multiple cellfates during early nervous system development. Dev Biol 338(1):50-62

10. Li B, Kuriyama S, Moreno M, Mayor R (2009) The posteriorizing gene Gbx2 is a direct target of Wnt signalling and the earliest factor in neural crest induction. Development 136(19):3267-3278

11. Nichane M, de Croze N, Ren X, Souopgui J, Monsoro-Burq AH, Bellefroid EJ (2008) Hairy2-Id3 interactions play an essential role in Xenopus neural crest progenitor specification. Dev Biol 322(2):355-367

12. Nichane M, Ren X, Souopgui J, Bellefroid EJ (2008) Hairy2 functions through both DNA-binding and non DNA-binding mechanisms at the neural plate border in Xenopus. Dev Biol 322(2):368-380

13. Maczkowiak F, Mateos S, Wang E, Roche D, Harland R, Monsoro-Burq AH (2010) The Pax3 and Pax7 paralogs cooperate in neural and neural crest patterning using distinct molecular mechanisms. Xenopus laevis embryos. Dev Biol 340(2):381-396

14. Basch ML, Bronner-Fraser M, Garcia-Castro MI (2006) Specification of the neural crest occurs during gastrulation and requires Pax7. Nature 441(7090):218-222

15. Otto A, Schmidt C, Patel K (2006) Pax3 and Pax7 expression and regulation in the avian embryo. Anat Embryol (Berl) 211(4):293-310

16. Khudyakov J, Bronner-Fraser M (2009) Comprehensive spatiotemporal analysis of early chick neural crest network genes. Dev Dyn 238(3):716-723

17. Phillips BT, Kwon HJ, Melton C, Houghtaling P, Fritz A, Riley BB (2006) Zebrafish msxB, msxC and msxE function together to refine the neural-nonneural border and regulate cranial placodes and neural crest development. Dev Biol 294(2):376-390

18. Esterberg R, Fritz A (2009) dlx3b/4b are required for the formation of the preplacodal region and otic placode through local modulation of BMP activity. Dev Biol 325(1):189-199

19. Kaji T, Artinger KB (2004) dlx $3 b$ and dlx $4 b$ function in the development of Rohon-Beard sensory neurons and trigeminal placode in the zebrafish neurula. Dev Biol 276(2):523-540

20. Klymkowsky MW, Rossi CC, Artinger KB (2010) Mechanisms driving neural crest induction and migration in the zebrafish and Xenopus laevis. Cell Adh Migr 4(4):595-608

21. Wang WD, Melville DB, Montero-Balaguer M, Hatzopoulos AK, Knapik EW (2011) Tfap2a and Foxd3 regulate early steps in the development of the neural crest progenitor population. Dev Biol 360(1):173-185

22. Artinger KB, Chitnis AB, Mercola M, Driever W (1999) Zebrafish narrowminded suggests a genetic link between formation of neural crest and primary sensory neurons. Development 126(18):3969-3979

23. Hernandez-Lagunas L, Choi IF, Kaji T, Simpson P, Hershey C, Zhou Y, Zon L, Mercola M, Artinger KB (2005) Zebrafish narrowminded disrupts the transcription factor prdm1 and is required for neural crest and sensory neuron specification. Dev Biol 278(2):347-357

24. John SA, Garrett-Sinha LA (2009) Blimp1: a conserved transcriptional repressor critical for differentiation of many tissues. Exp Cell Res 315(7):1077-1084

25. Inoue $\mathrm{T}$, Hatayama $\mathrm{M}$, Tohmonda $\mathrm{T}$, Itohara $\mathrm{S}$, Aruga $\mathrm{J}$, Mikoshiba K (2004) Mouse Zic5 deficiency results in neural tube defects and hypoplasia of cephalic neural crest derivatives. Dev Biol 270(1):146-162 
26. Inoue T, Ota M, Mikoshiba K, Aruga J (2007) Zic2 and Zic3 synergistically control neurulation and segmentation of paraxial mesoderm in mouse embryo. Dev Biol 306(2):669-684

27. Mitchell PJ, Timmons PM, Hebert JM, Rigby PW, Tjian R (1991) Transcription factor AP-2 is expressed in neural crest cell lineages during mouse embryogenesis. Genes Dev 5(1):105-119

28. Lyons GE, Houzelstein D, Sassoon D, Robert B, Buckingham ME (1992) Multiple sites of Hox-7 expression during mouse embryogenesis: comparison with retinoic acid receptor mRNA localization. Mol Reprod Dev 32(4):303-314

29. Steventon B, Carmona-Fontaine C, Mayor R (2005) Genetic network during neural crest induction: from cell specification to cell survival. Semin Cell Dev Biol 16(6):647-654

30. Patthey C, Edlund T, Gunhaga L (2009) Wnt-regulated temporal control of BMP exposure directs the choice between neural plate border and epidermal fate. Development 136(1):73-83

31. Steventon B, Araya C, Linker C, Kuriyama S, Mayor R (2009) Differential requirements of BMP and Wnt signalling during gastrulation and neurulation define two steps in neural crest induction. Development 136(5):771-779

32. Bonano M, Tribulo C, De Calisto J, Marchant L, Sanchez SS, Mayor R, Aybar MJ (2008) A new role for the Endothelin-1/ Endothelin-A receptor signaling during early neural crest specification. Dev Biol 323(1):114-129

33. Li W, Cornell RA (2007) Redundant activities of Tfap2a and Tfap $2 \mathrm{c}$ are required for neural crest induction and development of other non-neural ectoderm derivatives in zebrafish embryos. Dev Biol 304(1):338-354

34. Zhao Q, Eberspaecher H, Lefebvre V, De Crombrugghe B (1997) Parallel expression of Sox9 and Col2a1 in cells undergoing chondrogenesis. Dev Dyn 209(4):377-386

35. Kuhlbrodt K, Herbarth B, Sock E, Hermans-Borgmeyer I, Wegner M (1998) Sox10, a novel transcriptional modulator in glial cells. J Neurosci 18(1):237-250

36. Labosky PA, Kaestner KH (1998) The winged helix transcription factor Hfh2 is expressed in neural crest and spinal cord during mouse development. Mech Dev 76(1-2):185-190

37. Ciruna B, Rossant J (2001) FGF signaling regulates mesoderm cell fate specification and morphogenetic movement at the primitive streak. Dev Cell 1(1):37-49

38. Jiang R, Lan Y, Norton CR, Sundberg JP, Gridley T (1998) The Slug gene is not essential for mesoderm or neural crest development in mice. Dev Biol 198(2):277-285

39. Murray SA, Gridley T (2006) Snail family genes are required for left-right asymmetry determination, but not neural crest formation, in mice. Proc Natl Acad Sci USA 103(27):10300-10304

40. Raven CP, Kloos J (1945) Induction by medial and lateral pieces of the archenteron roof, with special reference to the determination of the neural crest. Acta Neerl Morphol 5:348-362

41. Mayor R, Morgan R, Sargent MG (1995) Induction of the prospective neural crest of Xenopus. Development 121(3):767-777

42. Bonstein L, Elias S, Frank D (1998) Paraxial-fated mesoderm is required for neural crest induction in Xenopus embryos. Dev Biol 193(2):156-168

43. Mancilla A, Mayor R (1996) Neural crest formation in Xenopus laevis: mechanisms of Xslug induction. Dev Biol 177(2):580-589

44. Marchant L, Linker C, Ruiz P, Guerrero N, Mayor R (1998) The inductive properties of mesoderm suggest that the neural crest cells are specified by a BMP gradient. Dev Biol 198(2):319-329

45. Rollhauser-ter Horst J (1979) Artificial neural crest formation in amphibia. Anat Embryol (Berl) 157(1):113-120

46. Moury JD, Jacobson AG (1990) The origins of neural crest cells in the axolotl. Dev Biol 141(2):243-253

47. Selleck MA, Bronner-Fraser M (1995) Origins of the avian neural crest: the role of neural plate-epidermal interactions. Development 121(2):525-538
48. Pieper M, Ahrens K, Rink E, Peter A, Schlosser G (2012) Differential distribution of competence for panplacodal and neural crest induction to non-neural and neural ectoderm. Development 139(6):1175-1187

49. Hong CS, Park BY, Saint-Jeannet JP (2008) Fgf8a induces neural crest indirectly through the activation of Wnt8 in the paraxial mesoderm. Development 135(23):3903-3910

50. Monsoro-Burq AH, Fletcher RB, Harland RM (2003) Neural crest induction by paraxial mesoderm in Xenopus embryos requires FGF signals. Development 130(14):3111-3124

51. Shi J, Severson C, Yang J, Wedlich D, Klymkowsky MW (2011) Snail2 controls mesodermal BMP/Wnt induction of neural crest. Development 138(15):3135-3145

52. Wu MY, Ramel MC, Howell M, Hill CS (2011) SNW1 is a critical regulator of spatial BMP activity, neural plate border formation, and neural crest specification in vertebrate embryos. PLoS Biol 9(2):e1000593

53. Ragland JW, Raible DW (2004) Signals derived from the underlying mesoderm are dispensable for zebrafish neural crest induction. Dev Biol 276(1):16-30

54. Dickinson ME, Selleck MA, McMahon AP, Bronner-Fraser M (1995) Dorsalization of the neural tube by the non-neural ectoderm. Development 121(7):2099-2106

55. Patthey C, Gunhaga L, Edlund T (2008) Early development of the central and peripheral nervous systems is coordinated by Wnt and BMP signals. PLoS ONE 3(2):e1625

56. Coffman CR, Skoglund P, Harris WA, Kintner CR (1993) Expression of an extracellular deletion of Xotch diverts cell fate in Xenopus embryos. Cell 73(4):659-671

57. Kengaku M, Okamoto H (1993) Basic fibroblast growth factor induces differentiation of neural tube and neural crest lineages of cultured ectoderm cells from Xenopus gastrula. Development 119(4):1067-1078

58. Basler K, Edlund T, Jessell TM, Yamada T (1993) Control of cell pattern in the neural tube: regulation of cell differentiation by dorsalin-1, a novel TGF beta family member. Cell 73(4): 687-702

59. Liem KF Jr, Tremml G, Roelink H, Jessell TM (1995) Dorsal differentiation of neural plate cells induced by BMP-mediated signals from epidermal ectoderm. Cell 82(6):969-979

60. Liem KF Jr, Tremml G, Jessell TM (1997) A role for the roof plate and its resident TGFbeta-related proteins in neuronal patterning in the dorsal spinal cord. Cell 91(1):127-138

61. Mayor R, Guerrero N, Martinez C (1997) Role of FGF and noggin in neural crest induction. Dev Biol 189(1):1-12

62. Saint-Jeannet JP, He X, Varmus HE, Dawid IB (1997) Regulation of dorsal fate in the neuraxis by Wnt-1 and Wnt-3a. Proc Natl Acad Sci USA 94(25):13713-13718

63. Chang C, Hemmati-Brivanlou A (1998) Neural crest induction by Xwnt7B in Xenopus. Dev Biol 194(1):129-134

64. LaBonne C, Bronner-Fraser M (1998) Neural crest induction in Xenopus: evidence for a two-signal model. Development 125(13): 2403-2414

65. Selleck MA, Garcia-Castro MI, Artinger KB, Bronner-Fraser M (1998) Effects of Shh and Noggin on neural crest formation demonstrate that BMP is required in the neural tube but not ectoderm. Development 125(24):4919-4930

66. Garcia-Castro MI, Marcelle C, Bronner-Fraser M (2002) Ectodermal Wnt function as a neural crest inducer. Science 297(5582):848-851

67. Kishigami S, Mishina Y (2005) BMP signaling and early embryonic patterning. Cytokine Growth Factor Rev 16(3):265278

68. Nie X, Luukko K, Kettunen P (2006) BMP signalling in craniofacial development. Int J Dev Biol 50(6):511-521

69. Massague J (2008) TGFbeta in cancer. Cell 134(2):215-230 
70. Tribulo C, Aybar MJ, Nguyen VH, Mullins MC, Mayor R (2003) Regulation of Msx genes by a Bmp gradient is essential for neural crest specification. Development 130(26):6441-6452

71. Nguyen VH, Schmid B, Trout J, Connors SA, Ekker M, Mullins MC (1998) Ventral and lateral regions of the zebrafish gastrula, including the neural crest progenitors, are established by a bmp2b/swirl pathway of genes. Dev Biol 199(1):93-110

72. Kwon HJ, Bhat N, Sweet EM, Cornell RA, Riley BB (2010) Identification of early requirements for preplacodal ectoderm and sensory organ development. PLoS Genet 6(9):e1001133

73. Tucker JA, Mintzer KA, Mullins MC (2008) The BMP signaling gradient patterns dorsoventral tissues in a temporally progressive manner along the anteroposterior axis. Dev Cell 14(1):108-119

74. Anderson RM, Stottmann RW, Choi M, Klingensmith J (2006) Endogenous bone morphogenetic protein antagonists regulate mammalian neural crest generation and survival. Dev Dyn 235(9):2507-2520

75. Faure S, de Santa Barbara P, Roberts DJ, Whitman M (2002) Endogenous patterns of BMP signaling during early chick development. Dev Biol 244(1):44-65

76. Stuhlmiller TJ, Garcia-Castro MI (2012) FGF/MAPK signaling is required in the gastrula epiblast for avian neural crest induction. Development 139(2):289-300

77. Sakai D, Suzuki T, Osumi N, Wakamatsu Y (2006) Cooperative action of Sox9, Snail2 and PKA signaling in early neural crest development. Development 133(7):1323-1333

78. Sakai D, Tanaka Y, Endo Y, Osumi N, Okamoto H, Wakamatsu Y (2005) Regulation of Slug transcription in embryonic ectoderm by beta-catenin-Lef/Tcf and BMP-Smad signaling. Dev Growth Differ 47(7):471-482

79. Schmid B, Furthauer M, Connors SA, Trout J, Thisse B, Thisse C, Mullins MC (2000) Equivalent genetic roles for bmp7/ snailhouse and bmp2b/swirl in dorsoventral pattern formation. Development 127(5):957-967

80. Kondo M (2007) Bone morphogenetic proteins in the early development of zebrafish. FEBS J 274(12):2960-2967

81. Beppu H, Kawabata M, Hamamoto T, Chytil A, Minowa O, Noda T, Miyazono K (2000) BMP type II receptor is required for gastrulation and early development of mouse embryos. Dev Biol 221(1):249-258

82. Fujiwara T, Dehart DB, Sulik KK, Hogan BL (2002) Distinct requirements for extra-embryonic and embryonic bone morphogenetic protein 4 in the formation of the node and primitive streak and coordination of left-right asymmetry in the mouse. Development 129(20):4685-4696

83. Gu Z, Reynolds EM, Song J, Lei H, Feijen A, Yu L, He W, MacLaughlin DT, van den Eijnden-van Raaij J, Donahoe PK, Li E (1999) The type I serine/threonine kinase receptor ActRIA (ALK2) is required for gastrulation of the mouse embryo. Development 126(11):2551-2561

84. Mishina Y, Crombie R, Bradley A, Behringer RR (1999) Multiple roles for activin-like kinase-2 signaling during mouse embryogenesis. Dev Biol 213(2):314-326

85. Mishina Y, Suzuki A, Ueno N, Behringer RR (1995) Bmpr encodes a type I bone morphogenetic protein receptor that is essential for gastrulation during mouse embryogenesis. Genes Dev 9(24):3027-3037

86. Dunn NR, Winnier GE, Hargett LK, Schrick JJ, Fogo AB, Hogan BL (1997) Haploinsufficient phenotypes in Bmp4 heterozygous null mice and modification by mutations in Gli3 and Alx4. Dev Biol 188(2):235-247

87. Kanzler B, Foreman RK, Labosky PA, Mallo M (2000) BMP signaling is essential for development of skeletogenic and neurogenic cranial neural crest. Development 127(5):1095-1104
88. Zhang H, Bradley A (1996) Mice deficient for BMP2 are nonviable and have defects in amnion/chorion and cardiac development. Development 122(10):2977-2986

89. Correia AC, Costa M, Moraes F, Bom J, Novoa A, Mallo M (2007) Bmp2 is required for migration but not for induction of neural crest cells in the mouse. Dev Dyn 236(9):2493-2501

90. Davis S, Miura S, Hill C, Mishina Y, Klingensmith J (2004) BMP receptor IA is required in the mammalian embryo for endodermal morphogenesis and ectodermal patterning. Dev Biol 270(1):47-63

91. Stottmann RW, Klingensmith J (2011) Bone morphogenetic protein signaling is required in the dorsal neural folds before neurulation for the induction of spinal neural crest cells and dorsal neurons. Dev Dyn 240(4):755-765

92. Dudas M, Sridurongrit S, Nagy A, Okazaki K, Kaartinen V (2004) Craniofacial defects in mice lacking BMP type I receptor Alk2 in neural crest cells. Mech Dev 121(2):173-182

93. Kaartinen V, Dudas M, Nagy A, Sridurongrit S, Lu MM, Epstein JA (2004) Cardiac outflow tract defects in mice lacking ALK2 in neural crest cells. Development 131(14):3481-3490

94. Stottmann RW, Choi M, Mishina Y, Meyers EN, Klingensmith J (2004) BMP receptor IA is required in mammalian neural crest cells for development of the cardiac outflow tract and ventricular myocardium. Development 131(9):2205-2218

95. Wang J, Nagy A, Larsson J, Dudas M, Sucov HM, Kaartinen V (2006) Defective ALK5 signaling in the neural crest leads to increased postmigratory neural crest cell apoptosis and severe outflow tract defects. BMC Dev Biol 6:51

96. Jia Q, McDill BW, Li SZ, Deng C, Chang CP, Chen F (2007) Smad signaling in the neural crest regulates cardiac outflow tract remodeling through cell autonomous and non-cell autonomous effects. Dev Biol 311(1):172-184

97. Ko SO, Chung IH, Xu X, Oka S, Zhao H, Cho ES, Deng C, Chai $Y$ (2007) Smad4 is required to regulate the fate of cranial neural crest cells. Dev Biol 312(1):435-447

98. Nie X, Deng CX, Wang Q, Jiao K (2008) Disruption of Smad4 in neural crest cells leads to mid-gestation death with pharyngeal arch, craniofacial and cardiac defects. Dev Biol 316(2):417-430

99. Choudhary B, Ito Y, Makita T, Sasaki T, Chai Y, Sucov HM (2006) Cardiovascular malformations with normal smooth muscle differentiation in neural crest-specific type II TGFbeta receptor (Tgfbr2) mutant mice. Dev Biol 289(2):420-429

100. Ito Y, Yeo JY, Chytil A, Han J, Bringas P Jr, Nakajima A, Shuler CF, Moses HL, Chai Y (2003) Conditional inactivation of Tgfbr2 in cranial neural crest causes cleft palate and calvaria defects. Development 130(21):5269-5280

101. Logan CY, Nusse R (2004) The Wnt signaling pathway in development and disease. Annu Rev Cell Dev Biol 20:781-810

102. Grigoryan T, Wend P, Klaus A, Birchmeier W (2008) Deciphering the function of canonical Wnt signals in development and disease: conditional loss- and gain-of-function mutations of beta-catenin in mice. Genes Dev 22(17):2308-2341

103. Sasai N, Mizuseki K, Sasai Y (2001) Requirement of FoxD3class signaling for neural crest determination in Xenopus. Development 128(13):2525-2536

104. Abu-Elmagd M, Garcia-Morales C, Wheeler GN (2006) Frizzled7 mediates canonical Wnt signaling in neural crest induction. Dev Biol 298(1):285-298

105. Deardorff MA, Tan C, Saint-Jeannet JP, Klein PS (2001) A role for frizzled 3 in neural crest development. Development 128(19):3655-3663

106. Hassler C, Cruciat CM, Huang YL, Kuriyama S, Mayor R, Niehrs C (2007) Kremen is required for neural crest induction in Xenopus and promotes LRP6-mediated Wnt signaling. Development 134(23):4255-4263 
107. Heeg-Truesdell E, LaBonne C (2006) Neural induction in Xenopus requires inhibition of Wnt-beta-catenin signaling. Dev Biol 298(1):71-86

108. Litsiou A, Hanson S, Streit A (2005) A balance of FGF, BMP and WNT signalling positions the future placode territory in the head. Development 132(18):4051-4062

109. Tamai K, Semenov M, Kato Y, Spokony R, Liu C, Katsuyama Y, Hess F, Saint-Jeannet JP, He X (2000) LDL-receptor-related proteins in Wnt signal transduction. Nature 407(6803):530-535

110. Villanueva S, Glavic A, Ruiz P, Mayor R (2002) Posteriorization by FGF, Wnt, and retinoic acid is required for neural crest induction. Dev Biol 241(2):289-301

111. Wu J, Yang J, Klein PS (2005) Neural crest induction by the canonical Wnt pathway can be dissociated from anterior-posterior neural patterning in Xenopus. Dev Biol 279(1):220-232

112. Carmona-Fontaine C, Acuna G, Ellwanger K, Niehrs C, Mayor $R$ (2007) Neural crests are actively precluded from the anterior neural fold by a novel inhibitory mechanism dependent on Dickkopf1 secreted by the prechordal mesoderm. Dev Biol 309(2):208-221

113. Lewis JL, Bonner J, Modrell M, Ragland JW, Moon RT, Dorsky RI, Raible DW (2004) Reiterated Wnt signaling during zebrafish neural crest development. Development 131(6):1299-1308

114. Wilson SI, Rydstrom A, Trimborn T, Willert K, Nusse R, Jessell TM, Edlund T (2001) The status of Wnt signalling regulates neural and epidermal fates in the chick embryo. Nature 411(6835):325-330

115. Skromne I, Stern CD (2001) Interactions between Wnt and Vg1 signalling pathways initiate primitive streak formation in the chick embryo. Development 128(15):2915-2927

116. Schmidt C, McGonnell IM, Allen S, Otto A, Patel K (2007) Wnt6 controls amniote neural crest induction through the noncanonical signaling pathway. Dev Dyn 236(9):2502-2511

117. Ikeya M, Lee SM, Johnson JE, McMahon AP, Takada S (1997) Wnt signalling required for expansion of neural crest and CNS progenitors. Nature 389(6654):966-970

118. Brault V, Moore R, Kutsch S, Ishibashi M, Rowitch DH, McMahon AP, Sommer L, Boussadia O, Kemler R (2001) Inactivation of the beta-catenin gene by Wnt1-Cre-mediated deletion results in dramatic brain malformation and failure of craniofacial development. Development 128(8):1253-1264

119. Tan C, Deardorff MA, Saint-Jeannet JP, Yang J, Arzoumanian A, Klein PS (2001) Kermit, a frizzled interacting protein, regulates frizzled 3 signaling in neural crest development. Development 128(19):3665-3674

120. Gray RS, Bayly RD, Green SA, Agarwala S, Lowe CJ, Wallingford JB (2009) Diversification of the expression patterns and developmental functions of the dishevelled gene family during chordate evolution. Dev Dyn 238(8):2044-2057

121. Dutta S, Dawid IB (2010) Kctd15 inhibits neural crest formation by attenuating Wnt/beta-catenin signaling output. Development 137(18):3013-3018

122. Wei S, Xu G, Bridges LC, Williams P, White JM, DeSimone DW (2010) ADAM13 induces cranial neural crest by cleaving class B Ephrins and regulating Wnt signaling. Dev Cell 19(2):345-352

123. Ossipova O, Sokol SY (2011) Neural crest specification by noncanonical Wnt signaling and PAR-1. Development 138(24):5441-5450

124. Bottcher RT, Niehrs C (2005) Fibroblast growth factor signaling during early vertebrate development. Endocr Rev 26(1):63-77

125. Dorey K, Amaya E (2010) FGF signalling: diverse roles during early vertebrate embryogenesis. Development 137(22):37313742

126. Fletcher RB, Baker JC, Harland RM (2006) FGF8 spliceforms mediate early mesoderm and posterior neural tissue formation in Xenopus. Development 133(9):1703-1714
127. Zhao H, Tanegashima K, Ro H, Dawid IB (2008) Lrig3 regulates neural crest formation in Xenopus by modulating Fgf and Wnt signaling pathways. Development 135(7):1283-1293

128. Lunn JS, Fishwick KJ, Halley PA, Storey KG (2007) A spatial and temporal map of FGF/Erk1/2 activity and response repertoires in the early chick embryo. Dev Biol 302(2):536-552

129. Hardy KM, Yatskievych TA, Konieczka J, Bobbs AS, Antin PB (2011) FGF signalling through RAS/MAPK and PI3K pathways regulates cell movement and gene expression in the chicken primitive streak without affecting E-cadherin expression. BMC Dev Biol 11:20

130. Karabagli H, Karabagli P, Ladher RK, Schoenwolf GC (2002) Comparison of the expression patterns of several fibroblast growth factors during chick gastrulation and neurulation. Anat Embryol (Berl) 205(5-6):365-370

131. Streit A, Berliner AJ, Papanayotou C, Sirulnik A, Stern CD (2000) Initiation of neural induction by FGF signalling before gastrulation. Nature 406(6791):74-78

132. Wilson SI, Graziano E, Harland R, Jessell TM, Edlund T (2000) An early requirement for FGF signalling in the acquisition of neural cell fate in the chick embryo. Curr Biol 10(8):421-429

133. Olivera-Martinez I, Storey KG (2007) Wnt signals provide a timing mechanism for the FGF-retinoid differentiation switch during vertebrate body axis extension. Development 134(11): 2125-2135

134. Furthauer M, Thisse C, Thisse B (1997) A role for FGF-8 in the dorsoventral patterning of the zebrafish gastrula. Development 124(21):4253-4264

135. Furthauer M, Van Celst J, Thisse C, Thisse B (2004) Fgf signalling controls the dorsoventral patterning of the zebrafish embryo. Development 131(12):2853-2864

136. Feldman B, Poueymirou W, Papaioannou VE, DeChiara TM, Goldfarb M (1995) Requirement of FGF-4 for postimplantation mouse development. Science 267(5195):246-249

137. Arman E, Haffner-Krausz R, Chen Y, Heath JK, Lonai P (1998) Targeted disruption of fibroblast growth factor (FGF) receptor 2 suggests a role for FGF signaling in pregastrulation mammalian development. Proc Natl Acad Sci USA 95(9):5082-5087

138. Meyers EN, Lewandoski M, Martin GR (1998) An Fgf8 mutant allelic series generated by Cre- and Flp-mediated recombination. Nat Genet 18(2):136-141

139. Sun X, Meyers EN, Lewandoski M, Martin GR (1999) Targeted disruption of Fgf8 causes failure of cell migration in the gastrulating mouse embryo. Genes Dev 13(14):1834-1846

140. Ciruna BG, Schwartz L, Harpal K, Yamaguchi TP, Rossant J (1997) Chimeric analysis of fibroblast growth factor receptor-1 (Fgfr1) function: a role for FGFR1 in morphogenetic movement through the primitive streak. Development 124(14):2829-2841

141. Deng CX, Wynshaw-Boris A, Shen MM, Daugherty C, Ornitz DM, Leder P (1994) Murine FGFR-1 is required for early postimplantation growth and axial organization. Genes Dev 8(24):3045-3057

142. Yamaguchi TP, Harpal K, Henkemeyer M, Rossant J (1994) fgfr-1 is required for embryonic growth and mesodermal patterning during mouse gastrulation. Genes Dev 8(24):3032-3044

143. Itoh $\mathrm{N}$ (2007) The Fgf families in humans, mice, and zebrafish: their evolutional processes and roles in development, metabolism, and disease. Biol Pharm Bull 30(10):1819-1825

144. Branney PA, Faas L, Steane SE, Pownall ME, Isaacs HV (2009) Characterisation of the fibroblast growth factor dependent transcriptome in early development. PLoS ONE 4(3):e4951

145. Fletcher RB, Harland RM (2008) The role of FGF signaling in the establishment and maintenance of mesodermal gene expression in Xenopus. Dev Dyn 237(5):1243-1254

146. Kudoh T, Concha ML, Houart C, Dawid IB, Wilson SW (2004) Combinatorial Fgf and Bmp signalling patterns the gastrula 
ectoderm into prospective neural and epidermal domains. Development 131(15):3581-3592

147. Kretzschmar M, Doody J, Massague J (1997) Opposing BMP and EGF signalling pathways converge on the TGF-beta family mediator Smad1. Nature 389(6651):618-622

148. Fuentealba LC, Eivers E, Ikeda A, Hurtado C, Kuroda H, Pera EM, De Robertis EM (2007) Integrating patterning signals: Wnt/ GSK3 regulates the duration of the BMP/Smad1 signal. Cell 131(5):980-993

149. Sapkota G, Alarcon C, Spagnoli FM, Brivanlou AH, Massague J (2007) Balancing BMP signaling through integrated inputs into the Smad1 linker. Mol Cell 25(3):441-454

150. Pera EM, Ikeda A, Eivers E, De Robertis EM (2003) Integration of IGF, FGF, and anti-BMP signals via Smad1 phosphorylation in neural induction. Genes Dev 17(24):3023-3028

151. Kuroda H, Fuentealba L, Ikeda A, Reversade B, De Robertis EM (2005) Default neural induction: neuralization of dissociated Xenopus cells is mediated by Ras/MAPK activation. Genes Dev 19(9):1022-1027

152. Andersson ER, Sandberg R, Lendahl U (2011) Notch signaling: simplicity in design, versatility in function. Development 138(17):3593-3612

153. Kuriyama S, Lupo G, Ohta K, Ohnuma S, Harris WA, Tanaka H (2006) Tsukushi controls ectodermal patterning and neural crest specification in Xenopus by direct regulation of BMP4 and X-delta-1 activity. Development 133(1):75-88

154. Endo Y, Osumi N, Wakamatsu Y (2002) Bimodal functions of Notch-mediated signaling are involved in neural crest formation during avian ectoderm development. Development 129(4):863873

155. Jiang YJ, Brand M, Heisenberg CP, Beuchle D, Furutani-Seiki M, Kelsh RN, Warga RM, Granato M, Haffter P, Hammerschmidt M, Kane DA, Mullins MC, Odenthal J, van Eeden FJ, Nusslein-Volhard C (1996) Mutations affecting neurogenesis and brain morphology in the zebrafish, Danio rerio. Development 123:205-216

156. Cornell RA, Eisen JS (2002) Delta/Notch signaling promotes formation of zebrafish neural crest by repressing Neurogenin 1 function. Development 129(11):2639-2648

157. Hernandez-Lagunas L, Powell DR, Law J, Grant KA, Artinger KB (2011) prdm1a and olig4 act downstream of Notch signaling to regulate cell fate at the neural plate border. Dev Biol 356(2):496-505

158. Filippi A, Tiso N, Deflorian G, Zecchin E, Bortolussi M, Argenton F (2005) The basic helix-loop-helix olig3 establishes the neural plate boundary of the trunk and is necessary for development of the dorsal spinal cord. Proc Natl Acad Sci USA 102(12):4377-4382

159. Yoon K, Gaiano N (2005) Notch signaling in the mammalian central nervous system: insights from mouse mutants. Nat Neurosci 8(6):709-715

160. De Bellard ME, Ching W, Gossler A, Bronner-Fraser M (2002) Disruption of segmental neural crest migration and ephrin expression in delta-1 null mice. Dev Biol 249(1):121-130

161. Bang AG, Papalopulu N, Kintner C, Goulding MD (1997) Expression of Pax-3 is initiated in the early neural plate by posteriorizing signals produced by the organizer and by posterior non-axial mesoderm. Development 124(10):2075-2085

162. Halilagic A, Zile MH, Studer M (2003) A novel role for retinoids in patterning the avian forebrain during presomite stages. Development 130(10):2039-2050

163. Uehara M, Yashiro K, Mamiya S, Nishino J, Chambon P, Dolle P, Sakai Y (2007) CYP26A1 and CYP26C1 cooperatively regulate anterior-posterior patterning of the developing brain and the production of migratory cranial neural crest cells in the mouse. Dev Biol 302(2):399-411
164. Aguero TH, Fernandez JP, Vega Lopez GA, Tribulo C, Aybar MJ (2012) Indian hedgehog signaling is required for proper formation, maintenance and migration of Xenopus neural crest. Dev Biol

165. Liem KF Jr, Jessell TM, Briscoe J (2000) Regulation of the neural patterning activity of sonic hedgehog by secreted BMP inhibitors expressed by notochord and somites. Development 127(22):4855-4866

166. Levine AJ, Brivanlou AH (2007) Proposal of a model of mammalian neural induction. Dev Biol 308(2):247-256

167. Elkouby YM, Frank D (2010) Wnt/ $\beta$-catenin signaling in vertebrate posterior neural development. Colloq Ser Dev Biol 1(1):1-79. doi:10.4199/C00015ED1V01Y201007DEB004

168. Delaune E, Lemaire P, Kodjabachian L (2005) Neural induction in Xenopus requires early FGF signalling in addition to BMP inhibition. Development 132(2):299-310

169. Linker C, Stern CD (2004) Neural induction requires BMP inhibition only as a late step, and involves signals other than FGF and Wnt antagonists. Development 131(22):5671-5681

170. Stavridis MP, Lunn JS, Collins BJ, Storey KG (2007) A discrete period of FGF-induced Erk1/2 signalling is required for vertebrate neural specification. Development 134(16):2889-2894

171. Stern CD (2005) Neural induction: old problem, new findings, yet more questions. Development 132(9):2007-2021

172. Roeser T, Stein S, Kessel M (1999) Nuclear beta-catenin and the development of bilateral symmetry in normal and LiCl-exposed chick embryos. Development 126(13):2955-2965

173. Wawersik S, Evola C, Whitman M (2005) Conditional BMP inhibition in Xenopus reveals stage-specific roles for BMPs in neural and neural crest induction. Dev Biol 277(2):425-442

174. Linker C, De Almeida I, Papanayotou C, Stower M, Sabado V, Ghorani E, Streit A, Mayor R, Stern CD (2009) Cell communication with the neural plate is required for induction of neural markers by BMP inhibition: evidence for homeogenetic induction and implications for Xenopus animal cap and chick explant assays. Dev Biol 327(2):478-486

175. Streit A, Lee KJ, Woo I, Roberts C, Jessell TM, Stern CD (1998) Chordin regulates primitive streak development and the stability of induced neural cells, but is not sufficient for neural induction in the chick embryo. Development 125(3):507-519

176. Lanner F, Rossant J (2010) The role of FGF/Erk signaling in pluripotent cells. Development 137(20):3351-3360

177. Kunath T, Saba-El-Leil MK, Almousailleakh M, Wray J, Meloche S, Smith A (2007) FGF stimulation of the Erk1/2 signalling cascade triggers transition of pluripotent embryonic stem cells from self-renewal to lineage commitment. Development 134(16):28952902

178. Di-Gregorio A, Sancho M, Stuckey DW, Crompton LA, Godwin J, Mishina Y, Rodriguez TA (2007) BMP signalling inhibits premature neural differentiation in the mouse embryo. Development 134(18):3359-3369

179. Nichols J, Silva J, Roode M, Smith A (2009) Suppression of Erk signalling promotes ground state pluripotency in the mouse embryo. Development 136(19):3215-3222

180. Greber B, Wu G, Bernemann C, Joo JY, Han DW, Ko K, Tapia N, Sabour D, Sterneckert J, Tesar P, Scholer HR (2010) Conserved and divergent roles of FGF signaling in mouse epiblast stem cells and human embryonic stem cells. Cell Stem Cell 6(3):215-226

181. Stavridis MP, Collins BJ, Storey KG (2010) Retinoic acid orchestrates fibroblast growth factor signalling to drive embryonic stem cell differentiation. Development 137(6):881-890

182. Sterneckert J, Stehling M, Bernemann C, Arauzo-Bravo MJ, Greber B, Gentile L, Ortmeier C, Sinn M, Wu G, Ruau D, Zenke M, Brintrup R, Klein DC, Ko K, Scholer HR (2010) Neural induction intermediates exhibit distinct roles of Fgf signaling. Stem Cells 28(10):1772-1781 
183. O'Rahilly R, Muller F (2007) The development of the neural crest in the human. J Anat 211(3):335-351

184. Thomas S, Thomas M, Wincker P, Babarit C, Xu P, Speer MC, Munnich A, Lyonnet S, Vekemans M, Etchevers HC (2008) Human neural crest cells display molecular and phenotypic hallmarks of stem cells. Hum Mol Genet 17(21):3411-3425

185. Betters E, Liu Y, Kjaeldgaard A, Sundstrom E, Garcia-Castro MI (2010) Analysis of early human neural crest development. Dev Biol 344(2):578-592

186. Pomp O, Brokhman I, Ben-Dor I, Reubinoff B, Goldstein RS (2005) Generation of peripheral sensory and sympathetic neurons and neural crest cells from human embryonic stem cells. Stem Cells 23(7):923-930

187. Fang D, Leishear K, Nguyen TK, Finko R, Cai K, Fukunaga M, Li L, Brafford PA, Kulp AN, Xu X, Smalley KS, Herlyn M (2006) Defining the conditions for the generation of melanocytes from human embryonic stem cells. Stem Cells 24(7):1668-1677

188. Lee G, Kim H, Elkabetz Y, Al Shamy G, Panagiotakos G, Barberi T, Tabar V, Studer L (2007) Isolation and directed differentiation of neural crest stem cells derived from human embryonic stem cells. Nat Biotechnol 25(12):1468-1475

189. Pomp O, Brokhman I, Ziegler L, Almog M, Korngreen A, Tavian M, Goldstein RS (2008) PA6-induced human embryonic stem cell-derived neurospheres: a new source of human peripheral sensory neurons and neural crest cells. Brain Res 1230:50-60

190. Chambers SM, Fasano CA, Papapetrou EP, Tomishima M, Sadelain M, Studer L (2009) Highly efficient neural conversion of human ES and iPS cells by dual inhibition of SMAD signaling. Nat Biotechnol 27(3):275-280

191. Jiang X, Gwye Y, McKeown SJ, Bronner-Fraser M, Lutzko C, Lawlor ER (2009) Isolation and characterization of neural crest stem cells derived from in vitro-differentiated human embryonic stem cells. Stem Cells Dev 18(7):1059-1070

192. Bajpai R, Chen DA, Rada-Iglesias A, Zhang J, Xiong Y, Helms J, Chang CP, Zhao Y, Swigut T, Wysocka J (2010) CHD7 cooperates with PBAF to control multipotent neural crest formation. Nature 463(7283):958-962

193. Curchoe CL, Maurer J, McKeown SJ, Cattarossi G, Cimadamore F, Nilbratt M, Snyder EY, Bronner-Fraser M, Terskikh AV (2010) Early acquisition of neural crest competence during hESCs neuralization. PLoS ONE 5(11):e13890

194. Lee G, Chambers SM, Tomishima MJ, Studer L (2010) Derivation of neural crest cells from human pluripotent stem cells. Nat Protoc 5(4):688-701

195. Valensi-Kurtz M, Lefler S, Cohen MA, Aharonowiz M, CohenKupiec R, Sheinin A, Ashery U, Reubinoff B, Weil M (2010) Enriched population of PNS neurons derived from human embryonic stem cells as a platform for studying peripheral neuropathies. PLoS ONE 5(2):e9290

196. Cimadamore F, Fishwick K, Giusto E, Gnedeva K, Cattarossi G, Miller A, Pluchino S, Brill LM, Bronner-Fraser M, Terskikh AV (2011) Human ESC-derived neural crest model reveals a key role for SOX2 in sensory neurogenesis. Cell Stem Cell 8(5):538-551

197. Menendez L, Yatskievych TA, Antin PB, Dalton S (2011) Wnt signaling and a Smad pathway blockade direct the differentiation of human pluripotent stem cells to multipotent neural crest cells. Proc Natl Acad Sci USA 108(48):19240-19245

198. Zhou Y, Snead ML (2008) Derivation of cranial neural crest-like cells from human embryonic stem cells. Biochem Biophys Res Commun 376(3):542-547

199. Christen B, Slack JM (1999) Spatial response to fibroblast growth factor signalling in Xenopus embryos. Development 126(1):119-125

200. Schohl A, Fagotto F (2002) Beta-catenin, MAPK and Smad signaling during early Xenopus development. Development 129(1):37-52

201. Revinski DR, Paganelli AR, Carrasco AE, Lopez SL (2010) Delta-Notch signaling is involved in the segregation of the three germ layers in Xenopus laevis. Dev Biol 339(2):477-492 